Pacific Journal of Mathematics

REAL ALGEBRAIC CURVES AND LINK COBORDISM 


\title{
REAL ALGEBRAIC CURVES AND LINK COBORDISM
}

\section{PATrick Gilmer}

\begin{abstract}
If $A$ is a nonsingular real algebraic curve of degree $m$, we show that there is a link cobordism of specified topological type between a link which depends only on the isotopy type of $A$ and a link which depends only on $m$. We prove a generalization of the TristramMurasugi inequalities for link cobordisms and apply it to this situation.
\end{abstract}

0. Introduction. In this paper, we will show that if a certain collection of simple closed curves, $C$, can be realized as a real algebraic curve of degree $m$ then there is a cobordism of specified topological type between two links in a 3-manifold $Q . Q$ may be described as $S^{3}$ modulo the quaternion eight group. One link, $L(C)$, depends only on $C$, and the other link depends only on $m$. This is our main result. It was announced at the Durham 4-manifolds conference in 1982. Since then it has been reformulated and improved with two addenda. Many of the known restrictions on link cobordisms in $S^{3}$ may be generalized to obtain restrictions on cobordisms in $Q$-homology 3-spheres. In [G3], we will develop the theory of 2-signatures and 2-nullities in a rational homology 3-sphere, and derive the generalized TristramMurasugi inequalities in this context. We will consider non-orientable as well as orientable cobordisms. Applying these results to the cobordism in $I \times Q$, we have obtained proofs of the strengthened Pretrovski inequalities, and the strengthened Arnold inequalities ((3.4), (3.5) (3.6) (3.7) (3.8) (3.9) of Viro's survey article [V]). Applying this same result to the lift of this cobordism to a covering space of $Q$, we have obtained Zvonilov's inequality ((3.23) of [V]). These derivations will appear in a sequel to this paper [G4]. Another result for links in $S^{3}$ states that the Arf invariants of two proper links in $S^{3}$, which are related by a planar cobordism, must be the same. The correct generalization of this result to links in a rational homology sphere will appear in [G3]. Applying these results to our cobordism in $Q$ and lifts of it to certain covers, we have rederived Rokhlin's congruence for $M$-curves, the related congruence for $M-1$ curves. (3.2) (3.3) [V] and Fiedler's congruence [F1]. These results will be included in [G4]. 
In $\S 1$, we explain the problem under investigation in extremely simple terms. In $\S 2$, we study the associated complex curve $C A$ and prove a theorem which allows us to guarantee certain curves are in "general position". In $\S 3$, we define $Q$ as the projective tangent circle bundle of $R P(2)$. We also define $L(A)$ and $L_{m}$. We also study $\widetilde{Q}$, the tangent circle bundle of $R P(2)$ and links which lie there. In this section we establish certain properties of these manifolds and links. In $\S 4$, we give a nice decomposition of $C P(2)$ which we use to prove our main theorem. Section 5 defines a simple invariant of surfaces with oriented boundary which we need for the statement of our theorem. In [G3], we will use this invariant to index our 2-signatures, and possible Arf invariants.

In $\S 6$, we state and prove our main Theorem (6.1). We also prove two addenda. Addenda (6.2) describes the cobordisms for collections of curves and may be used to study the topology of affine curves. Addenda (6.3) allows us to add cobordisms arising from certain auxiliary complex lines. We have tried to say as much as we can about the resulting cobordisms. Thus we prove more than is needed for the presently known corollaries. We do this in the hope that as more tools are developed for studying link cobordisms, it will be easier to apply them to the study of real algebraic curves. In $\S 7$, we give a new formulation of a theorem of Viro and Zvonilov's (4.8) of [V1] in terms of linking numbers. In $\S 8$, we use the Kirby calculus to describe $Q$ and the other spaces which arise in this paper. We describe $L(A)$ in a Kirby calculus picture of $Q$. This was the way we first described $L(A)$ in 1982 . We also give a Kirby calculus proof of the MasseyKuiper-Arnold theorem that $C P(2)$ modulo complex conjugation is the 4-sphere.

Section 9 presents Theorem (9.5), a generalization of the TristramMurasugi inequalities associated to odd prime powers. The ViroZvonilov inequalities (3.10) of [V] can be derived by applying this inequality to the lift of our cobordism to $I \times \widetilde{Q}$. We will give this derivation of the Viro-Zvonilov inequalities in [G4]. Theorem (9.7) gives a result of applying (9.5) to the cobordism in $I \times Q$. It is stated in terms of certain signatures of $L(C)$. At the moment, it is hard to apply this theorem as the calculation of the signatures of $L(A)$ is difficult. We are working on a new method which should make the calculation more manageable.

We have tried to be very careful about orientations. We orient a boundary using the last inward convention. 
1. The basic questions to be studied. We are concerned with the placement problem for a nonsingular real algebraic curve in the real projective plane. Let $F$ be a real homogeneous polynomial of degree $m$ in three variables $x_{0}, x_{1}, x_{2}$. The zero set of $F$ consists of a union of lines through the origin in $R^{3}$. The real projective plane $R P(2)$ is the set of lines through the origin in $R^{3}$ and thus the zero locus of $F$ is determined by a set $A$ in $R P(2)$ called the curve of $F . S^{2}$, the unit sphere about the origin in $R^{3}$, double covers $R P(2)$ and the inverse image of $A$ is the set of zeros of $F$ restricted to this sphere. $A$ is called nonsingular if the ordinary gradient $\nabla F$ in $R^{3}$ does not vanish on the zeros of $F$. Euler's equality (an easy exercise) says $\left(x_{0}, x_{1}, x_{2}\right) \cdot \nabla F=m F$. Therefore along $S^{2} \cap F^{-1}(0)$, $\nabla F$ is tangent to $S^{2}$. Thus a nonsingular curve $A$ is a smooth one dimensional submanifold of $R P(2)$. In other words $A$ is a collection of disjoint smooth simple closed curves in $R P(2)$.

A simple closed curve in $R P(2)$ is one of two types. It may separate $R P(2)$ into an open disk and an open Möbius band. In this case, it is called an oval and the disk is called the interior of the oval and the Möbius band is called the exterior of the oval. Otherwise it has a neighborhood homeomorphic to an open Möbius band and its complement is an open disk. We will call such a curve 1-sided. This follows immediately from the classification theorem for 2-manifolds. As a disk can contain no 1 -sided curves, $A$ can include at most one 1 -sided curve.

Consider $F$ on $S^{2}$ and shade the regions where $F$ is positive. If $m$ is even, then the antipodal regions on $S^{2}$ have the same shading and we can thus shade the corresponding regions in the complement of $A$ in $R P(2)$. Since $A$ must be the boundary of the shaded regions, $A$ does not contain a 1 -sided curve. Thus an even degree nonsingular curve consists entirely of ovals. If $m$ is odd, antipodal regions have the opposite sign. Join two antipodal points on $S^{2}$ not lying over $A$ by a path. The path projects to a loop which crosses $A$ an odd number of times. Thus $A$ must contain a 1 -sided curve. So for $m$ odd, $A$ consists of one 1-sided curve and a collection of ovals in the disk which is the complement of the 1 -sided curve.

The basic question is how can these ovals be arranged up to isotopy for a polynomial of given degree. As any two ovals are isotopic and any two 1-sided curves are isotopic, this question boils down to two questions: How many ovals can there be? What nesting arrangements can occur. We say one oval $O_{1}$ is nested in another oval $O_{2}$, if $O_{1}$ 
lies in the interior of $O_{2}$. In 1876, Harnack showed

$$
\beta_{0}(A) \leq 1+(1 / 2)(d-1)(d-2) .
$$

A curve with this number of components is called an $M$-curve. Harnack showed that there exist $M$-curves for all $m$. Hilbert in his sixteenth problem asked how the ovals of an $M$-curve can be arranged.

One may also ask how two or more curves can sit with respect to each other modulo ambient isotopy. For instance, we can ask how a curve of degree $m$ can sit with respect to a curve of degree one (a line). As a projective transformation will take any line to the line at infinity, we are really asking how affine curves can look.

If $A$ is a real algebraic curve given by $F$, we define $C A \subset C P(2)$ to be $\left\{\left[z_{0}, z_{1}, z_{2}\right] \mid F\left(z_{0}, z_{1}, z_{2}\right)=0\right\}$. If $A$ separates $C A$ into two components, $A$ is called dividing. If $A$ is dividing and nonsingular, it inherits an orientation as the boundary of the closure of one component of $C A-R A$. In the next section, we will see that we may assume $C A$ is a complex submanifold with a canonical orientation. This orientation on $R A$ is really a semi-orientation as it is only well defined up to changing the orientation on every component of $R A$ at once. This ambiguity comes from the arbitrary choice of one of the two components of $C A-R A$. This semi-orientation is called the complex orientation. This is an idea of Rokhlin's [R1], [R2]. Thus one can wonder whether a given isotopy class arises as a dividing and/or non-dividing curve. If it can arise as a dividing curve, what complex orientations can it have?

2. The complex curve $C A$. If $A$ is nonsingular, we can assume after changing the real coefficients a small amount that the complex curve $C A$ given by $F$ is nonsingular. This is an eminently reasonable statement, and some statement to this effect is made without proof in most papers on this subject. It is the purpose of this section to give a proof of a generalization of this statement. We also want to guarantee that $C A$ may be taken to be transverse to a given collection of other curves $\Gamma_{i}$ assuming $C A$ is already transverse to them along $A$. Along the way it is convenient to give a proof of the well-known fact that a nonsingular curve of degree $m$ in $C A$ has genus $(1 / 2)(m-1)(m-2)$. We will use this fact later. Thus if the above seems perfectly reasonable or familiar the reader may want to skip this section.

Let $W_{m}$ denote the vector space of all homogeneous polynomials of degree $m$ in $z_{0}, z_{1}$, and $z_{2}$ with complex coefficients. $W_{m}$ 
has a basis consisting of monomials of degree $m$. So $\operatorname{dim} W_{m}=$ $(1 / 2) m(m+3)$. Equations that differ by scalar multiples define the same zero set. Thus the associated projective space $P\left(W_{m}\right)$ can be thought of as the space of all equations for complex curves of degree $m$. We borrow the following proposition and proof from Namba [N] (1.4.3).

Proposition (2.1). $S=\left\{F \in P\left(W_{m}\right) \mid F\right.$ defines a singular curve $\}$ is a proper algebraic subset of $P\left(W_{m}\right)$.

Proof. An algebraic set is the zero of a set of homogeneous polynomials. Thus $\widetilde{S}=\left\{(p, F) \in C P(2) \times P\left(W_{m}\right) \mid \nabla F(p)=0\right.$ and $F(p)=0\}$ is an algebraic set. Let $\pi: C P(2) \times P\left(W_{m}\right) \rightarrow P\left(W_{m}\right)$ denote projection on the second factor. By the Main Theorem of elimination theory [M] $(2.23), \pi(\widetilde{S})=S$ is algebraic. $S$ is proper because there exist nonsingular curves of any degree, for instance the Fermat curves $F=z_{0}^{m}+z_{1}^{m}+z_{2}^{m}$. Note for this $F, \nabla F$ is never zero in $C^{3}-(0,0,0)$.

Corollary (2.2). $P\left(W_{m}\right)-S$ is path connected. Thus any two nonsingular curves are isotopic and therefore diffeomorphic as embedded oriented 2-manifolds.

Proof. Let $F_{1}, F_{2} \in P\left(W_{m}\right)-S$. Let $L$ be the line in $P\left(W_{m}\right)$ through $F_{1}$ and $F_{2} . L$ is a $C P(1)$ and $L \cap S$ is a proper algebraic set and thus a finite set of points. Clearly we can find a path in $L-S$ joining $F_{1}$ and $F_{2}$. This yields an isotopy between the curves.

COROLlary (2.3). The genus of any nonsingular curve of degree $m$ is $(1 / 2)(m-1)(m-2)$.

Proof. We only need to exhibit one with this genus for each $m$. Let $\pi: C P(2) \rightarrow C P(1) \approx S^{2}$ given by $\pi\left[z_{0}, z_{1}, z_{2}\right]=\left[z_{0}, z_{1}\right]$. When restricted to the Fermat curve, $\pi$ is easily seen to be an $m$-fold cyclic branched covering map with branching over $m$ points. If we triangulate $S^{2}$ with these $m$ points as vertices, then we can lift to a triangulation of the curve. A simple counting argument shows the Euler characteristic is $m \chi\left(S^{2}\right)-(m-1) m$ or $3 m-m^{2}$. Thus it has the above genus.

Let $U_{n}$ denote the vector space of all homogeneous polynomials in $z_{0}, z_{1}$, and $z_{2}$ with real coefficients, and $P\left(U_{n}\right)$ the associated real projective space. 
COROLlaRY (2.4). $S_{R}=S \cap P\left(U_{n}\right)$ is proper real algebraic set.

Proof. The real and imaginary parts of the equations which define $S$ in $P\left(W_{n}\right)$ define $S_{R}$.

Corollary (2.5). Suppose $F \in U_{n}$ defines a nonsingular $A$ in $R P(2)$. By changing the coefficients of $F$ an arbitrarily small amount, we find $F^{\prime}$ defining $A^{\prime}$ isotopic to $A$ and $C A^{\prime}$ nonsingular in $C P(2)$.

Proof. A proper real algebraic set is nowhere dense. This follows from the results stated in the beginning of $\S 2$ [Mi1].

In fact we will sometimes wish to insure that $C A$ be transverse to a collection of algebraic curves which are already transverse to $A$ in $R P(2)$. This can be done by adapting the proof of Prop. (1.4.4) of [N].

Proposition (2.6). Let $\Lambda_{i}$ be a finite collection of algebraic curves in $C P(2)$. Suppose $A$ intersects each $\Lambda_{i} \cap R P(2)$ transversely in $R P(2)$ at nonsingular points of $\Lambda_{i}$ which are not points of intersection with another $\Lambda_{j}$. Then we can change the coefficients of $F$ an arbitrarily small amount (keeping them real) so that $C A$ is nonsingular and $\Lambda_{i}$ and $C A$ intersect transversely without changing $A \cup \bigcup_{i}\left(\Lambda_{i} \cap R P(2)\right)$ up to ambient isotopy. Moreover we may insist that $C A$ avoids a finite number of given points $\left\{q_{j}\right\}$.

Proof. Let $\Lambda_{i}$ be defined by $G_{i}$. Let

$$
\begin{aligned}
Y_{i}=\left\{(p, F) \in C P(2) \times P\left(W_{n}\right) \mid F(p)=G_{i}(p)=0,\right. \\
\left.\left(\frac{\partial F}{\partial z_{j}}\right)\left(\frac{\partial G_{i}}{\partial z_{k}}\right)=\left(\frac{\partial F}{\partial z_{k}}\right)\left(\frac{\partial G_{i}}{\partial z_{j}}\right) \text { for } j=0,1,2\right\} .
\end{aligned}
$$

Define $\tilde{Y}$ to be the union of the $Y_{i}$. As a finite union of algebraic sets, $\widetilde{Y}$ is an algebraic set. Therefore $\pi(\widetilde{Y})=Y$ is algebraic. Thus $Z=Y \cup S$ is algebraic ( $S$ is from the proof of Proposition (2.1)). As above we conclude $Z_{R}$ is a real algebraic set in $P\left(U_{m}\right)$ and so nowhere dense. Thus we can vary the coefficients of $F$ an arbitrarily small amount to guarantee that $F$ is not in $Z_{R}$. If we add to the equations defining $\widetilde{S}$ the equations $F\left(q_{j}\right)=0$, then the new $C A$ will miss $\left\{q_{j}\right\}$. 
3. Two circle bundles over $R P(2)$. In this section, we discuss elementary properties of $S T R P(2)$, the tangent $S^{1}$ bundle of $R P(2)$ and $P T R P(2)$ the projective tangent bundle of $R P(2)$. We also define certain links in them which lie over curves in $R P(2)$. Later in this section, we will show the first space which we denote $\widetilde{Q}$ is diffeomorphic to $L(4,3)$. The second which we denote $Q$ is diffeomorphic to $S^{3}$ modulo the quaterions with some orientation. These facts have a long history. However they are not relevant to our main argument. See [S-T, p. 205] and [P] and the references therein. In (3.2), §4, §6, and $\S 8$, we will come back to these identifications, eventually taking into account orientations. For the time being, we wish to think of these spaces as circle bundles over $R P(2)$. Note the tangent bundle of a manifold has a canonical orientation given by a local orientation at a point followed by that same orientation in the fiber. Orient the tangent disk bundle of $R P(2)$ thus. This induces an orientation on $\widetilde{Q}$ and thus $Q$.

Given a collection on immersed transverse circles $C=\left\{C_{i}\right\}$ in $R P(2)$, we can assign an oriented link $\widetilde{L}(C)$ in $\widetilde{Q}$ and an unoriented link $L(C)$ in $Q$ as follows. We have:

$$
\begin{aligned}
& Q=\{(x, l) \mid x \in R P(2) l \text { a line in } R P(2) \text { through } x\}, \\
& \widetilde{Q}=\{(x, l) \mid x \in R P(2), l \text { an oriented line in } R P(2) \text { through } x\} .
\end{aligned}
$$

To each point on $C_{i}$, we may associate a point $x$ in $R P(2)$ and the line tangent to $C_{i}$ through $x$. This defines a knot $L\left(C_{i}\right)$ in $Q$ lying over $C_{i}$ in $R P(2) . L(C)$ is the link with components $L\left(C_{i}\right)$. Let $\widetilde{L}\left(C_{i}\right)$ be the 2-component link in $\widetilde{Q}$ obtained by considering both unit tangent vectors to $C_{i}$ at $x$. We let $\widetilde{L}(C)$ be the link in $\widetilde{Q}$ given by the union of $\widetilde{L}\left(C_{i}\right)$. Note $\widetilde{L}(C)$ has a natural orientation whether $C$ does or not. We oriented each component of $\widetilde{L}\left(C_{i}\right)$ by first orienting $C_{i}$ in the direction of the choice of unit tangent vector that the component corresponds to and then lifting the orientation to the component in question. If $C$ is oriented, then we may lift the orientation to $L(C)$ and obtain an oriented link. Finally if $C$ is oriented, we may define an oriented knot $L_{+}\left(C_{i}\right)$ in $\widetilde{Q}$ lying over $C_{i}$. One simply chooses the unit tangent vector in the direction of the orientation, and lifts the orientation from below. Again $L_{+}(C)$ is the union of $L_{+}\left(C_{i}\right)$.

We also associate a framing to the normal bundles of $L(C), \widetilde{L}(C)$ and $L_{+}(C)$ as follows. If $C_{i}$ has a trivial normal bundle draw a parallel curve $C_{i}^{\prime}$. If its normal bundle is 1-sided, we draw an almost 
parallel cure $C_{i}^{\prime}$ by perturbing the immersion by a section of the normal bundle of the immersion which is zero at only one point. Let $C^{\prime}$ denote this pushed off collection of curves. Frame $L\left(C_{i}\right), \widetilde{L}\left(C_{i}\right)$, and $L_{+}\left(C_{i}\right)$ so that its push-off is $L\left(C_{i}^{\prime}\right), \widetilde{L}\left(C_{i}^{\prime}\right)$ and $L_{+}\left(C_{i}^{\prime}\right)$ respectively. It is easy to see that this framing is well defined up to isotopy.

Let $X_{n}$ denote the nonorientable $D^{2}$ bundle over $R P(2)$ with Euler number $n$ and $S_{n}=\partial X_{n}$. We have $S_{1}=\widetilde{Q}$ and $S_{2}=Q$. Here $X_{n}$ has an orientation as a 4-manifold and the Euler number is $R P(2) \circ$ $R P(2)$ in $X_{n}$. Poincare duality and the long exact sequence of the pair $\left(X_{n}, S_{n}\right)$ give us:

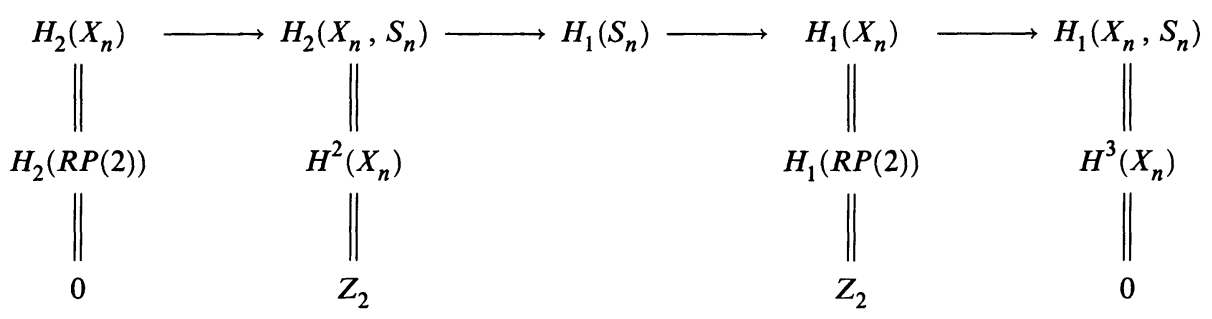

Thus $H_{1}\left(S_{n}\right)$ is either $Z_{2} \oplus Z_{2}$ or $Z_{4}$. Now consider the same diagram with $Z_{2}$ coefficients. The first horizontal map now between $Z_{2}$ and $Z_{2}$ is given by multiplication by $n \bmod 2$. Thus $H_{1}\left(S_{n}, Z_{2}\right)$ is either $Z_{2}$ if $n$ is odd and $Z_{2} \oplus Z_{2}$ if $n$ is even. Therefore $H_{1}\left(S_{n}\right)$ is $Z_{4}$ if $n$ is odd and $Z_{2} \oplus Z_{2}$ if $n$ is even.

If $n$ is odd, $H_{1}\left(S_{n}\right)$ is generated by any element which maps to the generator of $H_{1}\left(X_{n}\right)$. Thus $H_{1}(\widetilde{Q}) \approx Z_{4}$ is generated by $L_{+}(\mathfrak{L})$ where $\mathfrak{L}$ is an oriented line in $R P(2)$. Let $\mathscr{A}$ denote the antipodal map on $S_{n}$ which we note extents over $X_{n}$. Let $\overline{\mathfrak{L}}$ denote $\mathfrak{L}$ with the opposite orientation. Note $\mathscr{A}\left[L_{+}(\mathfrak{L})\right]$ is $L_{+}(\overline{\mathfrak{L}})$ with the opposite orientation, and $\mathfrak{L}$ and $\overline{\mathfrak{L}}$ are isotopic in $R P(2)$ (just spin $\mathfrak{L}$ $\left.180^{\circ}\right)$. Thus $L_{+}(\overline{\mathfrak{L}})$ and $L_{+}(\mathfrak{L})$ are isotopic. So we have $\mathscr{A}\left[L_{+}(\mathfrak{L})\right]=$ $-\left[L_{+}(\overline{\mathfrak{L}})\right]=-\left[L_{+}(\mathfrak{L})\right]$ and $\mathscr{A}$ induces on $H_{1}(\widetilde{Q})$ the map given by multiplication by minus one. Let $g$ denote $\left[L_{+}(\mathfrak{L})\right]$ in $H_{1}(\widetilde{Q})$.

If $n$ is even, $H_{1}\left(S_{n}\right)$ is generated by the fiber and a curve that maps to the generator of $H_{1}\left(X_{n}\right)$. Thus $H_{1}(Q) \approx Z_{2} \oplus Z_{2}$ is generated by $f$, the homology class of the fiber, and $b$, the homology class of $L(\mathfrak{L})$. The inverse image of $\mathfrak{L}$ under the projection is a Klein bottle $K_{\mathfrak{L}}$. By considering the action of $\mathscr{A}$ on $K_{\mathfrak{L}}$, one sees $\mathscr{A}_{\#}(f)=f$ and $\mathscr{A}_{\#}(b)=f+b$. We may define another involution of $Q$ using the duality between lines and points in $R P(2)$. Given a point $p=[a, b, c]$ of $R P(2)$, define $\mathfrak{T}(p)$ to be the line $a x_{0}+b x_{1}+c x_{2}=0$ in $R P(2)$. This gives a well-defined bijection between point and lines 
in $R P(2)$. Define $\mathscr{T}: Q \rightarrow Q$ by $\mathscr{T}(x, \mathfrak{L})=\left(\mathfrak{T}^{-1} \mathfrak{L}, \mathfrak{T} x\right)$. It is clear that $\mathscr{T}(f)=\mathscr{b}$ and $\mathscr{T}(b)=f$. Let $K_{p}$ be the Klein bottle $\mathscr{T}\left(K_{T(p)}\right)$. $K_{\mathfrak{L}}$ consists of all pairs: a point $q$ on $\mathfrak{L}$, a direction through $q . K_{p}$ consists of all pairs: a point $r$, the direction of the line through $r$ and $p$. Let $\langle$,$\rangle denote the intersection$ pairing $H_{1}\left(Q, Z_{2}\right) \times H_{2}\left(Q, Z_{2}\right) \rightarrow Z_{2}$; then we have $\left\langle f, K_{p}\right\rangle=$ $1,\left\langle f, K_{\mathfrak{L}}\right\rangle=0,\left\langle\mathscr{b}, K_{p}\right\rangle=\left\langle\mathscr{T} \mathfrak{b}, \mathscr{T} K_{p}\right\rangle=\left\langle f, K_{\mathfrak{L}}\right\rangle=0$, and $\left\langle\mathscr{b}, K_{\mathfrak{L}}\right\rangle=\left\langle\mathscr{T} \mathfrak{b}, \mathscr{T} K_{\mathfrak{L}}\right\rangle=\left\langle f, K_{p}\right\rangle=1$. It follows that $K_{\mathfrak{L}}$ and $K_{p}$ (for any choice of $\mathfrak{L}$ and $p$ ) represent generators for $H_{2}\left(Q, Z_{2}\right)$. We are also interested in the Bockstein $\beta: H_{2}\left(Q, Z_{2}\right) \rightarrow H_{1}(Q, Z)$. The Bockstein is natural so we only need to consider $\beta$ on the Klein bottles $K_{\mathfrak{L}}$ and $K_{p}$. Thus $\beta\left[K_{\mathfrak{L}}\right] \in H_{1}\left(K_{\mathfrak{L}}\right)$ is the unique 2-torsion class. In this way we see that $\beta\left[K_{\mathfrak{L}}\right]=f$ and $\beta\left[K_{p}\right]=b$ in $H_{1}(Q, Z)$. Note that $\mathscr{A}$ and $\mathscr{T}$ generate a subgroup of group of diffeomorphisms of $Q$ which is isomorphic to the symmetric group on three letters. The isomorphism is given by considering the action on the nonzero elements of $H_{1}(Q)$. Define $\alpha \in H^{1}\left(Q, Z_{2}\right)$ by $\alpha(f)=1$, and $\alpha(b)=0$. The 2 -fold cover $\widetilde{Q} \rightarrow Q$ is classified by $\alpha$.

REMARK (3.1). One can define the linking number of two disjoint knots in a rational homology sphere. This linking number may be rational. If the rational homology sphere is the boundary of a rational homology ball, then one can calculate linking numbers by counting the intersections between a pair of surfaces whose boundary is a multiple of the given link and dividing by the appropriate integer. Thus to calculate linking numbers in $S_{n}$, we may count intersections in $X_{n}$. If $O_{1}$ and $O_{2}$ are two disjoint, unnested ovals, then $\operatorname{lk}\left(L\left(O_{1}\right), L\left(O_{2}\right)\right)$ and $\operatorname{lk}\left(L_{+}\left(O_{1}\right), L_{+}\left(O_{2}\right)\right)$ is zero. If they are disjoint, nested and oriented in the same direction (so that they are homologous in the complement of a point in the interior of both of them), $\operatorname{lk}\left(L\left(O_{1}\right), L\left(O_{2}\right)\right)=2$ and $\operatorname{lk}\left(L_{+}\left(O_{1}\right), L_{+}\left(O_{2}\right)\right)=1$. If they are oriented in opposite directions $\operatorname{lk}\left(L\left(O_{1}\right), L\left(O_{2}\right)\right)=-2$ and $\operatorname{lk}\left(L_{+}\left(O_{1}\right), L_{+}\left(O_{2}\right)\right)=-1$. Suppose $\mathscr{L}$ is a 1 -sided curve and $O$ is an oval disjoint from $\mathscr{L}$ oriented in the same direction ( $O$ is homologous to twice $\mathscr{L}$ in the complement of a point in the interior of $C)$, then $\operatorname{lk}(L(O), L(\mathscr{L}))=1$ and $\operatorname{lk}\left(L_{+}(O), L_{+}(\mathscr{L})\right)=(1 / 2)$. If oriented in the opposite direction, then $\operatorname{lk}(L(O), L(\mathscr{L}))=-1$ and $\operatorname{lk}\left(L_{+}(O), L_{+}(\mathscr{L})\right)=-(1 / 2)$. The linking number of two fibers in $Q$ or $\widetilde{Q}$ is zero. Note that $\mathscr{T}$ sends the link consisting of two fibers in $Q$ to the link with the two components $L\left(\mathscr{L}_{1}\right)$ and $L\left(\mathscr{L}_{2}\right)$, where $\mathscr{L}_{1}$ and $\mathscr{L}_{2}$ are two lines in $R P(2)$. Thus $\operatorname{lk}\left(L\left(\mathscr{L}_{1}\right), L\left(\mathscr{L}_{2}\right)\right)$ is zero. 
On the other hand, one calculates that $\operatorname{lk}\left(L_{+}\left(\mathscr{L}_{1}\right), L_{+}\left(\mathscr{L}_{2}\right)\right)=-1 / 4$. Given a local orientation at a point $p$ in $R P(2)$, we may orient the fiber $f_{p}$ over that point in $Q$ or $\widetilde{Q}$, as the boundary of the disk fiber with this local orientation. Suppose $O$ encloses $p$ and is oriented as the boundary of its interior with the extension of the local orientation, then $\operatorname{lk}\left(L(O), f_{p}\right)=\operatorname{lk}\left(L_{+}(O), f_{p}\right)=1$.

REMARK (3.2). Rubenstein [Ru] has shown that a free $Z_{4}$ or $Q(8)$ action on $S^{3}$ is equivalent to an orthogonal action (his result is more general). As $S^{3}$ double covers $R P(3), R P(3)$ is diffeomorphic to $\mathrm{SO}(3)$ which is in turn diffeomorphic to $S T S^{2}$, and $S T S^{2}$ double covers $\operatorname{STR} P(2)$, we see that $S^{3}$ is an 8-fold cover of $Q$ and a 4-fold cover of $\widetilde{Q}$. As $Q(8)$ is the only group of order eight with first homology $Z_{2} \oplus Z_{2}$ and $Z_{4}$ is the only group of order four with first homology $Z_{4}$, we conclude that $Q$ is indeed $S^{3}$ modulo a free orthogonal action of $Q(8)$ and that $\widetilde{Q}$ is $S^{3}$ modulo an orthogonal action of $Z_{4}$. Thus $\widetilde{Q}$ is $L(4,1)$ or $L(4,3)$. As $\operatorname{lk}\left(L_{+}\left(\mathscr{L}_{1}\right), L_{+}\left(\mathscr{L}_{2}\right)\right)=-1 / 4, \widetilde{Q}$ must be $L(4,3)$. The next section will incidently give another proof of this last fact. Price [P], and Rubenstein [Ru] showed that the mapping class group of $Q$ is $S_{3}$ and Rubenstein showed that the mapping class group of $L(4,1)$ is $Z_{2}$. Thus we have realized all the elements in terms of $\mathscr{A}$ and $\mathscr{T}$.

4. A decomposition of $C P(2)$. In this section, we show how $C P(2)$ can be divided into three pieces. One piece is diffeomorphic to $I \times$ $L(4,3)$ and the other two are tubular neighborhoods of $R P(2)$ and $\Sigma$. Here $\Sigma$ denotes the quadratic Fermat curve $x_{0}^{2}+x_{1}^{2}+x_{2}^{2}=0$ which is a 2 -sphere missing $R P(2)$. Let $h: C P(2) \rightarrow[0,1]$ be given by

$$
h\left[z_{0}, z_{1}, z_{2}\right]=\frac{\left|z_{0}^{2}+z_{1}^{2}+z_{2}^{2}\right|}{\left|z_{0}\right|^{2}+\left|z_{1}\right|^{2}+\left|z_{2}\right|^{2}} .
$$

By the triangle inequality, $|h| \leq 1$. Moreover $h\left[z_{0}, z_{1}, z_{2}\right]=1$ if and only if $z_{0}^{2}, z_{1}^{2}$ and $z_{2}^{2}$ all lie in the same direction (i.e. are all positive real multiples of each other). This means $h^{-1}(1)=R P(2)$.

THEOREM (4.1). $h^{2}$ is a $C^{\infty}$ generalized Morse function on $C P(2)$ with two critical submanifolds: $h^{-1}(1)=R P(2)$ of index 2 and $h^{-1}(1)=\Sigma$ of index 0 . 
Proof. See Bott [B, p. 344] or Hirsh [H, p. 149 exercise 6 which should read "partial tubular neighborhood"] for definitions. Consider

$$
\hat{h}^{2}\left[z_{0}, z_{1}, z_{2}\right]=\frac{\left(z_{0}^{2}+z_{1}^{2}+z_{2}^{2}\right)\left(\overline{z_{0}^{2}}+\overline{z_{1}^{2}}+\overline{z_{2}^{2}}\right)}{\left(z_{0} \overline{z_{0}}+z_{1} \overline{z_{1}}+z_{2} \overline{z_{2}}\right)^{2}} .
$$

It is easy to see that $\hat{h}^{2}$ is $C^{\infty}$ on $C^{3}-(0,0,0)$ and induces $h^{2}$ on $C P(2)$. It is elementary but tedious to check that $\nabla \hat{h}^{2} \neq 0$ in $C^{3}-(0,0,0)$ except above $R P(2)$ and $\Sigma$. We have

$$
h^{2}[a i, b i, 1]=\left(\frac{1-a^{2}-b^{2}}{1+a^{2}+b^{2}}\right)^{2}
$$

and

$$
\hat{h}^{2}((1, i, 0)+(a+b i)(1,-1,0))=\frac{4\left(a^{2}+b^{2}\right)}{\left(1+a^{2}+b^{2}\right)^{2}} .
$$

These show that the Hessian on the normal fibers is nondegenerate with given index.

REMARK. We used this function to prove an early version of (6.1) in 1982. Fiedler has independently studied and used this same function [F2]. He thanks V. Kharlamov for pointing out the function to him.

We now decompose $C P(2)$ into three pieces: $h^{-1}[0, \delta]$, $h^{-1}[\delta, 1-\delta]$, and $h^{-1}[1-\delta, 0]$. Here $\delta$ denotes a small number. The second is diffeomorphic to a product $I \times h^{-1}(\delta)$ by $[\mathbf{H}$, (6.2.2)] or [Mi2]. The first and third are diffeomorphic to the closed disk bundles associated to the normal bundles of $\Sigma$ and $R P(2)$ in $C P(2)$, by the exercise in $[\mathbf{H}]$ referred to above. Since $\Sigma \circ \Sigma=4$, and $\Sigma$ is orientable, the normal bundle $\nu_{\Sigma}$ is an $\mathrm{SO}(2)$ bundle with Euler number 4 . Thus we see that $h^{-1}(\delta)$, oriented as the boundary of $\nu_{\Sigma}$, and therefore $h^{-1}(t)$, oriented as the boundary of $h^{-1}[0, t]$ (a submanifold of $C P(2)$ with its complex orientation) for $t \in(0,1)$, are diffeomorphism to $L(4,3)$.

On the other end of the product, we identify $h^{-1}(1-\delta)$ with $\widetilde{Q}$, the $S^{1}$ bundle of tangent bundle of $R P(2)$. Multiplication by $i$ takes tangent vectors to $R P(2)$ to normal vectors to $R P(2)$. This defines $J$, an isomorphism of $O(2)$-bundles between $T R P(2)$ and $\nu_{R P(2)}$ has an orientation as a 4-manifold induced from the orientation obtained from the complex structure on $C P(2)$ and the diffeomorphism of the total space of $\nu_{R P(2)}$ with a tubular neighborhood. The total space of $T R P(2)$ has a natural orientation obtained by choosing some local orientation of $R P(2)$ around a point together with this same orientation of the fiber over this point. The map $J$ induces an orientation 
reversing map on the total spaces. Thus we see the map $J$ induces an orientation preserving diffeomorphism of $\widetilde{Q}$ with $h^{-1}(1-\delta)$ oriented as the boundary of $h^{-1}[0,1-e]$, a submanifold of $C P(2)$ with its complex orientation. Moreover the Euler number of the tangent bundle, i.e. $R P(2) \circ R P(2)$ in $T R P(2)$, is $\chi(R P(2))=1$. Therefore $R P(2) \circ R P(2)$ in $C P(2)$ is -1 . See Wilson [W, (2.4)].

REMARK (4.2). We can give a nice explicit identification of $C P(2)-$ $\Sigma$ with the open tangent disk bundle of $R P(2)$. Let $E=\{(v, w) \mid$ $v, w \in R^{3},|w|<|v|=1$ and $\left.v \cdot w=0\right\}$ denote the open unit tangent disk bundle of $S^{2}$. The quotient $\bar{E}$ of $E$ by the involution sending $(v, w)$ to $(-v,-w)$ is the open unit tangent disk bundle of $R P(2)$. Define $\psi: E \rightarrow C P(2)$ by $\psi\left(\left(v_{0}, v_{1}, v_{2}\right),\left(w_{0}, w_{1}, w_{2}\right)\right)=$ $\left[v_{0}+i w_{0}, v_{1}+i w_{1}, v_{2}+i w_{2}\right]$. Note each point in $C P(2)-\Sigma$ has a representative $z=\left(z_{0}, z_{1}, z_{2}\right)$ with $z \cdot z=z_{0}^{2}+z_{1}^{2}+z_{2}^{2}$ real and greater than zero. Let $v, w \in R^{3}$ with $z=v+i w$. Note $z \cdot z=v \cdot v-w \cdot w+2 v \cdot w i$. So $v \cdot v>w \cdot w$ and $v \cdot w=0$. There are exactly two such representations $\pm z$ with $v \cdot v=1$. So we see $\psi$ is a $2-1$ map onto $C P(2)-\Sigma$ and induces a diffeomorphism $\bar{\psi}$ of $\bar{E}$ with $C P(2)-\Sigma$ sending the zero section $R P(2)$ identically onto $R P(2)$. Notice $h \bar{\psi}(v, w)=\left(1-|w|^{2}\right) /\left(1+|w|^{2}\right)$. So the level sets $h^{-1}(\delta)$ correspond to the sphere bundles of given length.

REMARK (4.3). This decomposition has a little extra structure. $\mathrm{SO}(3)$ acts on $C^{3}-0$ and thus $C P(2)$ by matrix multiplication. It is easy to check that $h$ is $\mathrm{SO}(3)$ invariant and it follows that the orbits of this $\mathrm{SO}(3)$ action are precisely the manifolds $\hat{h}^{-1}(t)$ for $t \in[0,1]$.

5. Surfaces with oriented boundary. In this section, we introduce a simple invariant of a surface with oriented boundary. It is a good bookkeeping device and has simplified our treatment of link cobordism.

Let $G$ be a possibly disconnected compact surface whose boundary has a given orientation. If $G$ is connected and without boundary, we define $\gamma(G)=\beta[G]$ in $H_{1}(G, Z)$. Here $[G]$ is the fundamental class in $H_{2}\left(G, Z_{2}\right)$ and $\beta$ is the Bochstein associated to the coefficient sequence $0 \rightarrow Z \rightarrow Z \rightarrow Z_{2} \rightarrow 0$. Equivalently, if $G$ is orientable $\gamma(G)=0$ and if not $\gamma(G)$ is the unique 2-torsion class in $H_{1}\left(G, Z_{2}\right)$. However the relation to the Bochstein is useful. If $G$ is connected with boundary, then $H_{1}(G)$ is free abelian and $[\partial G] \in H_{1}(G)$, defined using the given orientation on $\partial G$, is null homologous modulo two. Define $\gamma(G)$, in this case to be $(1 / 2)[\partial G] \in H_{1}(G)$. In general, define 
$\gamma(G)$ to be $\bigoplus \gamma\left(G_{i}\right)$ where the $G_{i}$ are the connected components of $G$ and $H_{1}(G)=\oplus H_{1}\left(G_{i}\right)$.

We can give a uniform definition as follows. Triangulate $G$ extending a triangulation of $\partial G$ if $\partial G$ is nonempty, with each 2-simplex meeting $\partial G$ in at most one edge. Choose orientations for the 2simplices, inducing the given orientation on the boundary. Then the boundary of the oriented 2-chain given by these simplices is a 1-chain representing $[\partial G]$ and twice another 1-chain $g$ which must be a 1cycle. We define $\gamma(G)$ to be the class represented by $g$. The following propositions are easy exercises.

Proposition (5.1). If $G^{\prime}$ and $G^{\prime \prime}$ are two surfaces with oriented boundaries and $G$ is formed by identifying some of the boundary components in an orientation reversing manner, then $\gamma(G)=i_{*}^{\prime} \gamma\left(G^{\prime}\right)+$ $i_{*}^{\prime \prime} \gamma\left(G^{\prime \prime}\right)$ where $i^{\prime}$ and $i^{\prime \prime}$ are inclusions of $G^{\prime}$ and $G^{\prime \prime}$ in $G$.

Proposition (5.2). If $G$ is a surface with oriented boundary, and $G^{\prime}$ is the same surface with the orientation on a component $K$ of $\partial G$ reversed, then $\gamma\left(G^{\prime}\right)=\gamma(G)-[K]$. Here $[K]$ is the homology class of $K$ as originally oriented.

Now if we have a map $f: G \rightarrow X$ and an orientation on $\partial G$, we will let $\gamma(G)$ denote $f_{*}(\gamma(G)$ ) as well. It will be clear from context where $\gamma(G)$ lives. Of course if $G$ is closed we will then have $\gamma(G)=$ $\beta([G])$ where now $\beta: H_{2}\left(X, Z_{2}\right) \rightarrow H_{1}(X, Z)$.

Let $\pi: \widetilde{X} \rightarrow X$ be a $d$-sheeted covering space projection. Define tr: $C_{n}(X) \rightarrow C_{n}(\tilde{X})$ by sending a simplex to the sum of the simplices covering it. It induces a well-defined map called the transfer $\operatorname{Tr}: H_{n}(X) \rightarrow H_{n}(\widetilde{X})$. One has $\pi_{*} \circ \operatorname{Tr}$ is multiplication by $d$. We have the following propositions whose proof we leave to the reader.

Proposition (5.3). If $\pi: \widetilde{G} \rightarrow G$ is a finite covering, and $\pi$ preserves orientations on $\partial \widetilde{G}$ and $\partial G$, then $\gamma(\widetilde{G})=\operatorname{Tr}(\gamma(G))$.

Proposition (5.4). If $\pi: \widetilde{X} \rightarrow X$ is a finite covering with $\widetilde{G} \subseteq \widetilde{X}$ and $G \subseteq X$, and $\pi$ preserves orientations on $\partial \widetilde{G}$ and $\partial G$, then $\gamma(\widetilde{G})=\operatorname{Tr}(\gamma(G))$.

6. Some link cobordisms. We let $\mathfrak{L}_{m}$ denote some collection of $m$ distinct lines in $R P(2)$ and let $L_{m}$ denote $L\left(\mathfrak{L}_{m}\right)$. Under the diffeomorphism $\mathscr{T}, L_{m}$ is sent to $m$ fibers. Clearly this new link 
is independent up to isotopy of the choice of fibers. Moreover we may move a fiber along an orientation reversing path in $R P(2)$ and obtain an isotopy of the oriented link $L_{m}$ to the link where the string orientation has been reversed on any one component. Thus the link $L_{m}$ is well defined up to isotopy. Moreover as an oriented link it is equivalent up to isotopy to itself with any choice of string orientation.

Let $M$ be an oriented 3-manifold. Let $i_{1}: M \rightarrow \partial(I \times M)$ be given by $i_{1}(m)=(1, m)$ and $i_{0}: M \rightarrow \partial(I \times M)$ be given by $i_{0}(m)=$ $(0, m)$. Then $i_{1}$ is orientation preserving and $i_{0}$ is orientation reversing. A properly embedded surface $G$ in $I \times M$ is said to be a cobordism from the link $\left(i_{1}\right)^{-1}(\partial G)$ in $M$ to the link $\left(i_{0}\right)^{-1}(\partial G)$ in $M$. Here is our main theorem.

THEOREM (6.1). If $A$ is a nonsingular real algebraic curve of degree $m$ with $\mathfrak{C}$ ovals, then there is a connected cobordism $G$ in $I \times Q$ from $L(A)$ to $L_{m}$ such that

(1) $\chi(G)=\left(m-m^{2}\right) / 2$.

(2) $j^{*} \alpha=w_{1}(G)$. Here $j$ denotes the inclusion of $G$ in $I \times Q$ composed with the projection to $Q$.

(3) $G \circ G=\left[m^{2} / 2\right]$. Here $G$ is pushed off itself in $I \times Q$ so that along $L(A)$ and $L_{m}$, it has been pushed off with their given framings, and [ ] denotes the greatest integer function.

(4) For any orientation of $L(A)$ and some orientation of $L_{m}, j_{*} \gamma(G)$ $=(\mathfrak{C}+[m / 2]) f$.

(5) $A$ is dividing if and only if $G$ is orientable. If $G$ is orientable, it induces the same orientation on $L(A)$ as the complex orientation on $A$ does.

(6) The projection on I has no local minima on the interior of $G$.

Addendum (6.2). If $\left\{A_{i}\right\}_{i=1}^{n}$ are nonsingular real algebraic curves of degree $m_{i}$ and each pair of curves $\left\{A_{i}, A_{j}\right\}$ intersects transversely in say $r_{i, j}$ points, then we have a collection of transverse cobordisms $G_{j}$ in $I \times Q$ satisfying (6.1) (where $m_{i}$ replaces $m$ and the lines appearing in all the collections $\mathfrak{L}_{m_{i}}$ are distinct). Moreover $G_{i}$ intersects $G_{j}$ transversely in $\left(m_{i} m_{j}-r_{i, j}\right) / 2$ points.

Given a line $\mathfrak{L}$ in $R P(2)$, define $D(\mathfrak{L})$ to be the curve obtained by taking two lines very close to $\mathfrak{L}$ with one to either side and resolving them at the point of intersection to make an embedded curve, as in Figure 1. We call $D(\mathfrak{L})$ the double of $\mathfrak{L}$. If we have a local orientation at a point $p$ of $R P(2)$, and a collection of oriented immersed circles 


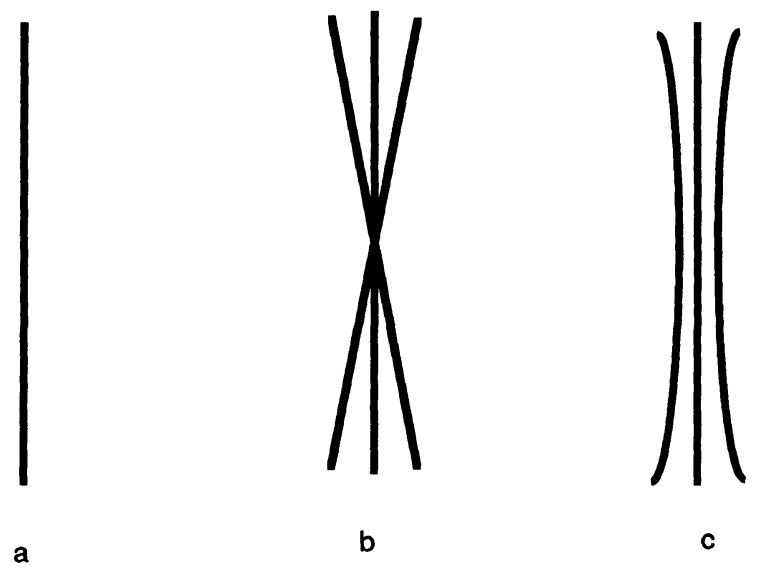

FIGURE 1

$C$ in $R P(2)-p$, we define $r(C, p)$, the rotation number of $C$ around $p$, as follows. Note that $H_{1}(R P(2)-p)$ is infinite cyclic. Let a small circle around $p$ be oriented as the boundary of its interior oriented with the extension of the local orientation. It represents twice some generator. Define $r(C, p)$ to be one half the multiple of this generator which is represented by $C$.

Addendum (6.3). We may also add to this collection of cobordisms further cobordisms as follows. Pick a collection of points $\left\{p_{k}\right\}$ in $R P(2)$ not on any $A_{i}$. Let $C_{k}$ denote a small circle around each $p_{k}$. Then there are annular cobordisms $\left\{H_{k}\right\}$ from $L\left(C_{k}\right)$ to $L\left(D_{k}\right)$. Here $D_{k}$ is a "double" of a line $\mathfrak{L}^{k}$, where the lines $\left\{\mathfrak{L}^{k}\right\}$ are distinct from each other and from the lines appearing in the $\mathfrak{L}_{m_{i}}$. For each $k, H_{k} \circ H_{k}=0$, when we push off along the boundaries with the given framings. For $k \neq k^{\prime} \quad H_{k}$ intersects $H_{k^{\prime}}$ in two points but with algebraic intersection number zero. Each $H_{k}$ intersects $G_{i}$ transversely in $m_{i}$ points. Pick a local orientation at $p_{k}$, orient $C_{k}$ as the boundary of its interior with this local orientation, and then orient $H_{k}$ so that it induces the same orientation on $L\left(C_{k}\right)$ as $C_{k}$ does. If $A_{i}$ is dividing and is given one of its complex orientations, then $G_{i} \circ H_{k}$ is $2 r\left(A_{i}, p_{k}\right)$.

Comments. Recall $\mathfrak{L}_{m}$ with any orientation is isotopic to $\mathfrak{L}_{m}$ with any other, so the qualification "some" in (4) is not significant. Also $\mathfrak{L}_{m}$ has no natural orientation arising from the problem, unless $A$ is dividing, in which case by (5) with this orientation $\gamma(G)$ is zero. By (5.2), if we change the orientation of one component of $\mathfrak{L}_{m}$, we change $\gamma(G)$ by $b$. Thus (4) says as much as we can hope for. 
The idea for Addendum (6.3) came from Fiedler's paper [F3] where he makes use of auxiliary complex lines in $C P(2)$. The idea for Addendum (6.1) comes from Viro's paper [V2] and Fiedler's paper [F4] where they make use of auxiliary real lines. The theorem itself was reported at the Durham 4-manifolds conference in 1982. At that time the links $L(A)$ were described by how they sat in a Kirby calculus picture of $Q$. See $\S 8$.

Proof (of (6.1)). By Proposition (2.6), we can assume that $C A$ is nonsingular and intersects $\Sigma$ transversely. By Proposition (2.3), $\chi(C A)=2-2 g$ where $g$ stands for $(1 / 2)(m-1)(m-2)$. So for $\delta$ small enough $C A \cap h^{-1}[0, \delta]$ is isotopic to $2 m$ disk fibers of the bundle $h^{-1}[0, \delta] \rightarrow \Sigma$.

If $v$ is a tangent vector to $A$ in $R P(2)$, then $i v$ is a tangent vector to $C A$ as $C A$ is a complex submanifold. But $i v$ is also a normal vector to $R P(2)$ as above. Thus we see that $C A \cap h^{-1}[1-\delta, 1]$ is isotopic to $J(T A)$ in $J(T R P(2)) \approx \nu_{R P(2)} \approx h^{-1}[1-\delta, 1]$ for $\delta$ small. Therefore $C A \cap h^{-1}(1-\delta)$ is $\widetilde{L}(A)$ in $\widetilde{Q}$. Here $h^{-1}(1-\delta)$ is oriented as the boundary of $h^{-1}[\delta, 1-\delta]$ which in turn inherits the orientation on $C P(2)$.

Let $T$ denote the involution on $C P(2)$ defined by $T\left[z_{0}, z_{1}, z_{2}\right]=$ $\left[\overline{z_{0}}, \overline{z_{1}}, \overline{z_{1}}\right]$. As $F$ has real coefficients, $T$ leaves $C A$ invariant. $T$ induces the map multiplication by -1 on $H_{2}(C P(2))$, and so is orientation reversing when restricted to $C A$. If $A$ is dividing, $C A-A$ has two components: say $C A_{ \pm}$. Then $T$ induces an orientation reversing diffeomorphism between $C A_{+}$and $C A_{-}$. Let $\widetilde{G}$ denote the intersection of $C A$ with $h^{-1}[\delta, 1-\delta]$. We have $\chi(\widetilde{G})=\chi(C A)-2 m=m-m^{2} . \widetilde{G}$ has a natural orientation coming from the complex structure on $C A$. If $C$ is dividing, $\widetilde{G}$ has two components diffeomorphic to each other. Let $G_{+}$be one of them. If $C$ is not dividing, $\widetilde{G}$ is connected and $T$ induces an orientation reversing free involution on $\widetilde{G}$.

We must see what curve a fiber of $h^{-1}(\delta) \rightarrow \Sigma$ is when viewed in $h^{-1}(1-\delta) \approx \widetilde{Q}$ under the diffeomorphism given by the product structure or equivalently the flow induced by $\nabla h^{2}$. Consider $C A$ above in the case $m=1$. We may as well choose $F\left[x_{0}, x_{1}, x_{2}\right]=x_{0}$. Then $C A$ intersects $\Sigma$ transversely at the two points $[0, \pm i, 1]$ and $C A \cap R P(2)$ is a line $\mathfrak{L}$. This is a dividing curve. Choose $C A_{+}$passing through $[0, i, 1]$. Give $\mathfrak{L}$ the resulting complex orientation it obtains from $C A_{+}$, and let $G_{+}$be $C A_{+} \cap G$. Then $G_{+}$is a cobordism in 
$h^{-1}[\delta, 1-\delta]$ between the fiber and $L_{+}(\mathfrak{L})$. We can parameterize $C A_{+}$by $\psi(u, v)=[0, u+i v, 1]$ for $u, v \in R$, and $v>0$. Note $h^{2} \psi(u, v)=1-\left(4 v^{2}\right) /\left(1+u^{2}+v^{2}\right)^{2}$. One can check that $\nabla h^{2} \circ \psi$ is non-zero except at $(0,1)$ which maps to $[0, i, 1] \in \Sigma$. Thus the cobordism $G_{+}$is level preserving and yields an isotopy of knots in $\widetilde{Q}$.

In general if $X \subseteq C P(2)$ is $T$-invariant, we will let $\bar{X}$ denote the orbit space of $X$ under $\left.T\right|_{X}$. Since the fix point set of $T$ is $R P(2)$, $C P(2)$ is the 2-fold branched cyclic cover of $\overline{C P(2)}$ along $\overline{R P(2)}$ and $\overline{R P(2)} \circ \overline{R P(2)}$ is $-2 .\left.T\right|_{\Sigma}$ is orientation reversing and fix point free. So $\bar{\Sigma} \approx R P(2)$ and $\bar{\Sigma} \circ \bar{\Sigma}$ is 2 , as $\Sigma \circ \Sigma$ is 4 .

$Q$ can be identified with $\overline{h^{-1}(1-\delta)}$ as both are orbit spaces of $\widetilde{Q}$ under the same free involution on $\widetilde{Q}$ classified by $\alpha \in H^{1}\left(Q, Z_{2}\right)$. As $h$ is invariant with respect to $T$, we see $\overline{h^{-1}[\delta, 1-\delta]}$ $\approx[\delta, 1-\delta] \times Q$. Let $G$ denote $\overline{\widetilde{G}}$. Then $G$ is embedded in $[\delta, 1-\delta] \times Q, G \cap\{1-\delta\} \times Q=L(C)$. We have $\chi(G)$ is one half $\chi(\widetilde{G})$. Since $\widetilde{G}$ is orientable, $w_{1}(G)$ is $\alpha$ restricted to $G$. Moreover $G$ is orientable if and only if $\widetilde{G}$ is disconnected if and only if $A$ is dividing. Finally $G \cap \overline{h^{-1}(\delta)}$ is $m$ fibers of the $S^{1}$-bundle $\overline{h^{-1}(\delta)} \rightarrow \bar{\Sigma}$.

As $\bar{\Sigma}=R P(2)$, the associated bundle is a rational homology ball. Thus the linking numbers of distinct fibers must be zero. So $G \cap \overline{h^{-1}(\delta)}$ is isomorphic to the link obtained from one fiber by shaking off $m-1$ extra components with mutual linking numbers zero. $L_{m}$ can be obtained from $L(\mathfrak{L})$ in just this way. The above isotopy between a fiber of $h^{-1}(\delta) \rightarrow \Sigma$ when viewed in $\delta \times \widetilde{Q}$ and $L_{+}(\mathfrak{L})$ gives us an isotopy between a fiber of $\overline{h^{-1}(\delta)} \rightarrow \bar{\Sigma}$ when viewed in $\delta \times Q$ and $L(\mathfrak{L})$. This is because $G_{+}$and $T\left(G_{+}\right)$are disjoint in the dividing $(m=1)$ case. It follows that $G \cap \overline{h^{-1}(\delta)}$ when viewed in $\delta \times Q$ is isotopic to $L_{m}$.

If we standardize with an affine map from $[\delta, 1-\delta]$ to $[0,1]=I$, we have proved our theorem with conditions: (1), (2), and (5). To prove (3), begin by letting $G_{0}$ be a pushoff of $G$ in $[\delta, 1-\delta] \times Q$ with the assigned framings at the ends. Let $\widetilde{G}_{0}$ be the surface lying over $G_{0}$ in $[\delta, 1-\delta] \times \widetilde{Q}$. $\widetilde{G}_{0}$ can be extended to a surface $C A_{0}$ in $C P(2)$ homologous to $C A$ as follows. In $h^{-1}[0, \delta]$, we can cap off $\bar{G}_{0}$ with disk fibers disjoint from $C A$. In $h^{-1}[1-\delta, 1]$, we add annuli corresponding to $T A_{0}$ where $A_{0}$ is the parallel push-off of $A$ used to define the framing of $\widetilde{L}(A)$. Thus if $m$ is odd, $C A$ and $C A_{0}$ 
will intersect in one point in $h^{-1}[1-\delta, 1]$ and if $m$ is even they will not intersect in this region. Thus we see that

$$
m^{2}=C A \circ C A_{0}=2\left(G \circ G_{0}\right)+ \begin{cases}1 & \text { if } m \text { is odd } \\ 0 & \text { if } m \text { is even }\end{cases}
$$

Since $G \circ G_{0}$ is our definition of $G \circ G$, (3) follows.

To prove (4) give $A$ and $\mathfrak{L}_{m}$ any orientation. Then $L(A)$ and $L\left(\mathfrak{L}_{m}\right)$ are oriented and $\gamma(G)$ is defined. If we give $\widetilde{G}$ the orientation it inherits from $C A$ and then orient $\widetilde{L}(A)$ and $\widetilde{L}\left(\mathfrak{L}_{m}\right)$ as the boundary, we get the natural orientation on $\widetilde{L}(A)$ and the opposite of the natural orientation on $\widetilde{L}\left(\mathfrak{L}_{m}\right)$. Next note that in order to make the covering map $\widetilde{G} \rightarrow G$ preserve orientations on the boundary, we must change the natural orientation on $\widetilde{L}(A)$ and $\widetilde{L}\left(\mathfrak{L}_{m}\right)$ by changing the orientation of exactly one curve lying over each component of $A$ and $\mathfrak{L}_{m}$. Let $\widetilde{G}_{1}$ denote $\widetilde{G}$ with the boundary oriented so that the covering to $G$ preserves orientation on the boundary, let $\widetilde{G}_{2}$ denote $\widetilde{G}$ with the natural orientations on $\widetilde{L}(A)$ and $\widetilde{L}\left(\mathfrak{L}_{m}\right)$, and $\widetilde{G}_{3}$ denote $\widetilde{G}$ with the boundary oriented as the boundary of $\widetilde{G}$ with the orientation induced from $C A$. We have $\gamma\left(\widetilde{G}_{3}\right)$ is zero. By $(5.2) \gamma\left(\widetilde{G}_{2}\right)=2 m g$, and

$$
\gamma\left(\widetilde{G}_{1}\right)=\gamma\left(\widetilde{G}_{2}\right)-m g-2 \mathfrak{C}_{g}- \begin{cases}g & \text { if } m \text { is odd } \\ 0 & \text { if } m \text { is even }\end{cases}
$$

By (5.3), $\gamma\left(\widetilde{G}_{1}\right)=\operatorname{Tr}(\gamma(G))$. Thus

$$
\operatorname{Tr}(\gamma(G))=([m / 2]-\mathfrak{C}) 2 g=([m / 2]+\mathfrak{C}) 2 g .
$$

Since $\operatorname{Tr} f=2 g$ and $\operatorname{Tr}(b)=0$, we conclude that $\gamma(G)=$ $([m / 2]+\mathfrak{C}) f+\mu b$ where $\mu$ is zero or one. However if $\mu$ is one, we simply change the orientation on one component of $L\left(\mathfrak{L}_{m}\right)$ and then $\gamma(G)$ is as stated in (4).

We only have the proof of (6) left. Let $p$ be a point of $G$ and assume without loss of generality that $p$ is in the chart given by $z_{0} \neq 0$. Thus $p=[1, a, b]$ and $\left[1, w_{1}, w_{2}\right]$ parameterizes a neighborhood of $p$. In these local coordinates, $G$ is given by $w_{1}=f_{1}(z)$ and $w_{1}=f_{2}(z)$, where $z \in U \subseteq C$ is a local complex coordinate for $C A$ around $p$ and the $f_{i}$ are complex analytic. Then $\left.(1 / h)\right|_{G}$ is given 
locally by

$$
\begin{aligned}
& \frac{1+\left|f_{1}(z)\right|^{2}+\left|f_{2}(z)\right|^{2}}{\left|1+\left(f_{1}(z)\right)^{2}+\left(f_{2}(z)\right)^{2}\right|} \\
& \quad=\left|\frac{1}{\sqrt{1+\left(f_{1}(z)\right)^{2}+\left(f_{2}(z)\right)^{2}}}\left(1, f_{1}(z), f_{2}(z)\right)\right|^{2} \\
& \quad=|H(z)|^{2}
\end{aligned}
$$

where $\sqrt{1}$ is a branch of the square root around $1+a^{2}+b^{2}$, $\left|\left(z_{0}, z_{1}, \ldots, z_{n}\right)\right|=\left|z_{0}\right|^{2}+\left|z_{1}\right|^{2}+\cdots\left|z_{n}\right|^{2}$, and $H: U \rightarrow C^{3}$ is defined by the above equation. Note that $H$ is analytic in each coordinate. We need the lemma given below which we could not find in the literature. If $\left.h\right|_{G}$ has a local minima, then $\left.(1 / h)\right|_{G}$ has a local maximum. Thus $|H(z)|$ has a local maximum. By Lemma (6.4), $H$ and thus $f_{1}$ and $f_{2}$ are constant, but this is impossible. This completes the proof of (6.1).

Lemma (6.4). If $U \subset C, H: U \rightarrow C^{n}$ is analytic in each coordinate, and $|H|$ has a local maximum, the $H$ is constant.

Proof. The lemma for $n=1$ is the familiar maximum modulus principle. For $n>1$, assume $|H|$ has a local maximum at $p$. Define $\widehat{H}(z)=H(z) \cdot \overline{H(p)}$. By the Cauchy-Schwarz inequality $|\widehat{H}(z)| \leq$ $|H(z)||\overline{H(p)}| \leq|H(p)|^{2}=\widehat{H}(p)$. As $\widehat{H}$ has a maximum at $p$, by the maximum modulus principle, $\widehat{H}$ is constant. So the Cauchy-Schwarz inequality must be an equality. Therefore $H(z)=\lambda(z) H(p)$ where $|\lambda(z)| \leq 1$ and $\lambda$ is analytic. As $\lambda(p)=1$, again by the maximum modulus principle $\lambda$ is constant. Therefore $H$ is constant.

Proof (of Addendum (6.2)). Using (2.6), we may inductively make $C A_{i}$ nonsingular, transverse to each other and to $\Sigma$, with no triple intersections. $C A_{i}$ intersects $C A_{j}$ positively in $m_{i} m_{j}$ points with $a_{i j}$ points occurring on $R P(2)$. The other points come in complex conjugate pairs away from $\Sigma$. The result follows.

Proof (of Addendum (6.3)). Consider the two lines $\mathfrak{L}_{ \pm i}$ through $[1,0,0]: z_{2}= \pm i z_{1}$. They intersect $\Sigma$ in one point $[0,1, \pm i]$. The individual intersections with $R P(2)$ are transverse at $[1,0,0]$. The intersections at $[0,1, \pm i]$ are tangencies of order two, because $\Sigma$ has degree two and $\mathfrak{L}_{ \pm i}$ have degree one. If we take a small $\delta$ neighborhood of $[0,1, \pm i]$ in $R P(2)$ and let $D_{\delta}$ denote the normal 


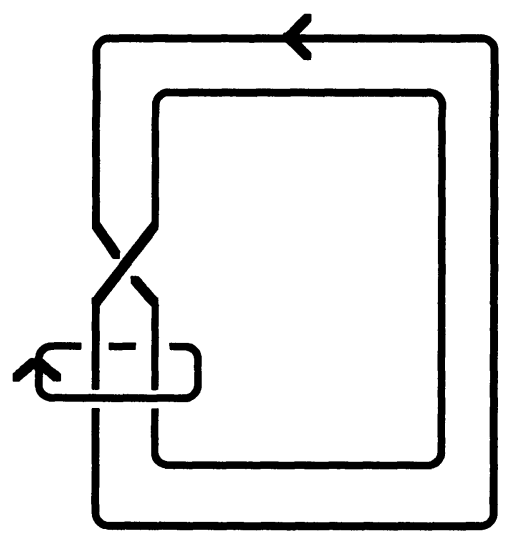

FIGURE 2

2-disk bundle over it in $h^{-1}[0, \delta]$, then $\Sigma \cap D_{\delta}$ (the 0-section) intersects $\partial D_{\delta}$ an unknot. Also $\mathfrak{L}_{ \pm i}$ will intersect $\partial D_{\delta}$ in an unknot lying in $h^{-1}(\delta)$. Thus we obtain a link in $\partial D_{\delta}$ with two unknotted components and with linking number 2 (as the multiplicity of the intersection is two). Here we orient the components as the boundary of $D_{\delta} \cap \Sigma$ and $D_{\delta} \cap \mathfrak{L}_{ \pm i}$. This link is an algebraic link given by the singularity $w\left(z^{2}-w^{2}-2 w\right)=0$. (Here we use the local chart: $z_{0}=z, z_{1}=1, z_{2}=i(w+1)$.) Thus it is a particular type of iterated torus link [E-N]. The only algebraic link with two unknotted components with linking number two is the symmetric link in Figure 2.

Note $\mathfrak{L}_{ \pm i}$ form two complex conjugate lines in $C P(2)$ and thus lead to a single annulus in $\overline{h^{-1}[\delta, 1-\delta]} \approx Q \times I$. We wish to describe $\overline{\mathfrak{L}_{i} \cap h^{-1}(\delta)}$ and $\overline{\mathfrak{L}_{i} \cap h^{-1}(1-\delta)}$ in $Q$. Consider the following process. Begin with the oriented circle fiber of $h^{-1}(\delta) \rightarrow \Sigma$ which maps to $[0,1, \pm i]$ and shake off two parallel fibers. Next join these fibers by a band sum where the band's core is the path of a point during the shake-off, and such that linking of the original fiber with the new curve is +1 (in either $\overline{\partial D_{\delta}}$ or $\overline{h^{-1}(\delta)}$ ). The linking numbers in $\overline{\partial D_{\delta}}$ or $\overline{h^{-1}(\delta)}$ will be the same as both can be computed from intersections in $\overline{D_{\delta}}$ as $\overline{D_{\delta}}$ is part of the rational ball $h^{-1}[0, \delta]$. See Figure 3 for the above process taking place in $\overline{\partial D_{\delta}}$. Thus we obtain in this way $\overline{\mathfrak{L}_{i} \cap h^{-1}(\delta)}$ up to an isotopy in $\overline{\partial D_{\delta}} \cap \overline{h^{-1}(\delta)}$.

Since a fiber of $\overline{h^{-1}(\delta)}$ corresponds to $L(\mathfrak{L})$ for some $\mathfrak{L}$ in $R P(2)$ under the diffeomorphism to $Q$ induced by the gradient of $h^{2}$, if we perform the same process to $L(\mathfrak{L})$ in $Q$, we will obtain $\overline{\mathfrak{L}_{i} \cap(0 \times Q)}$ 

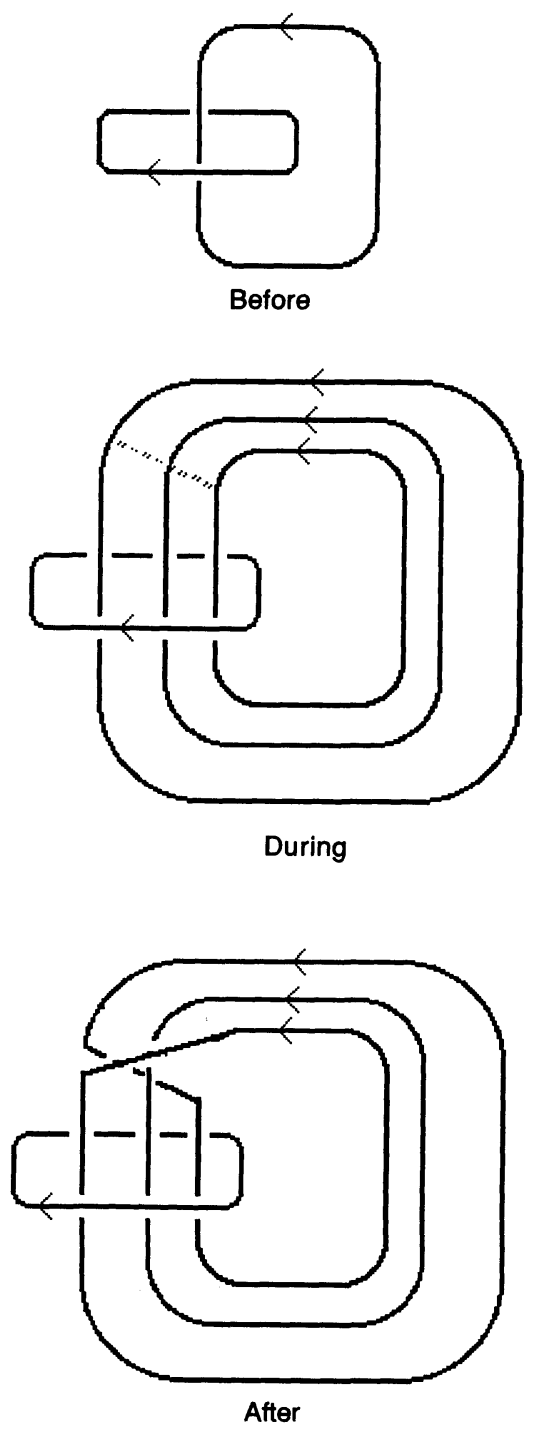

FIGURE 3

in $Q$. By (3.1), $L k(L(\mathfrak{L}), L(D(\mathfrak{L})))$ is one. Moreover $L(D(\mathfrak{L}))$ arises from a band sum of the two pushed off lines along a band with the specified core. Thus we see that $\overline{\mathfrak{L}_{i} \cap(0 \times Q)}$ in $Q$ is given by $L(D(\mathfrak{L}))$.

Now $\mathfrak{L}_{ \pm}$intersects $R P(2)$ transversely at $[1,0,0]$. They also intersect each other transversely there. Take a small $\delta$-neighborhood $D_{\delta}$ of $[1,0,0]$ in $R P(2)$ and let $B_{\delta}$ denote the normal 2-disk bundle over it in $h^{-1}[1-\delta, 1]$. Then $\mathfrak{L}_{ \pm}$and $R P(2)$ intersect $\partial B_{\delta}$ in a link with three unknotted components. Pick a local orientation of 
$R P(2)$ in a neighborhood of $[1,0,0]$, say that given by the chart $x_{0}=1, x_{1}=x$, and $x_{2}=y$, and the orientation on $R^{2}$ given by the frame $e_{1}, e_{2}$. Then orient $\partial B_{\delta}$ as the boundary of $B_{\delta}$ with the orientation it inherits from $C P(2)$, and orient the components of the link as the boundary of the oriented disks they bound in $B_{\delta}$. Complex conjugation induces an orientation preserving involution on $\partial B_{\delta}$ which fixes the component on $R P(2)$ and switches the other two components while reversing their string orientations. An elementary exercise using stereographic projection allows one to see this situation clearly. Up to isotopy the oriented link is given in Figure 4a. One may also see the 2-component link in the orbit space $\overline{\partial B_{\delta}}$. We use the orientation on the intersection with $\mathfrak{L}_{i}$ to orient one component of the link in the quotient. It is pictured in Figure $4 \mathrm{~b}$. We have identified $\overline{h^{-1}(1-\delta)}$ oriented as the boundary of $h^{-1}[1-\delta, 1]$ with $Q=P T R P(2)$ in an orientation reversing way. Note $\overline{\partial B_{\delta}} \cap \overline{h^{-1}(1-\delta)}$ is sent to $P T D_{\delta}$ by a bundle map (they are both circle bundles over $D_{\delta}$ ) which is orientation reversing on the fibers. We are interested in the knot $\overline{\mathfrak{L}_{i}} \cap 1 \times Q$ in $1 \times Q$ oriented as the boundary of $\overline{\mathfrak{L}_{i}} \cap I \times Q$ with $\overline{\mathfrak{L}_{i}}$ oriented with the orientation of the complex manifold $\mathfrak{L}_{i}$. This lies in the boundary of the disk bundle associated to $P T D_{\delta}$. We may obtain a picture of it here from Figure $4 \mathrm{~b}$. We should reflect the circle fibers across a horizontal plane and reverse the orientation of the component associated to $\overline{\mathfrak{L}_{i}}$. In this way we obtain Figure $4 \mathrm{c}$, and after an isotopy we arrive at Figure $4 \mathrm{~d}$. This can be described as a picture of $P T C_{\delta}$ in $P T D_{\delta}$ where $C_{\delta}$ is a small circle around $[1,0,0]$ oriented as the boundary of its interior with the local orientation at $[1,0,0]$.

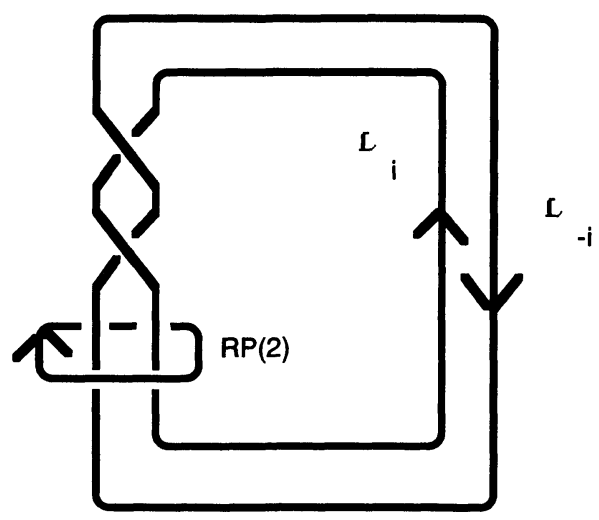

FIGURE 4a

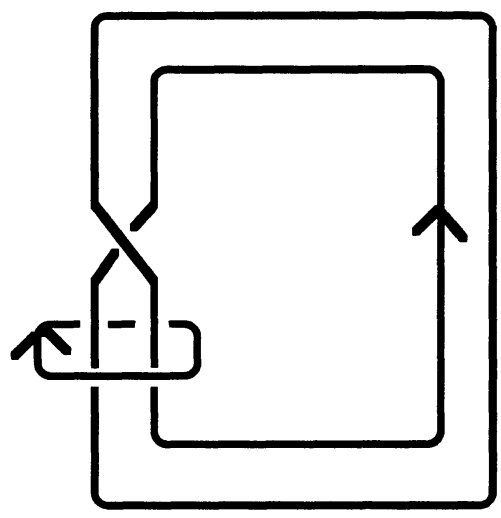

Figure 4b 


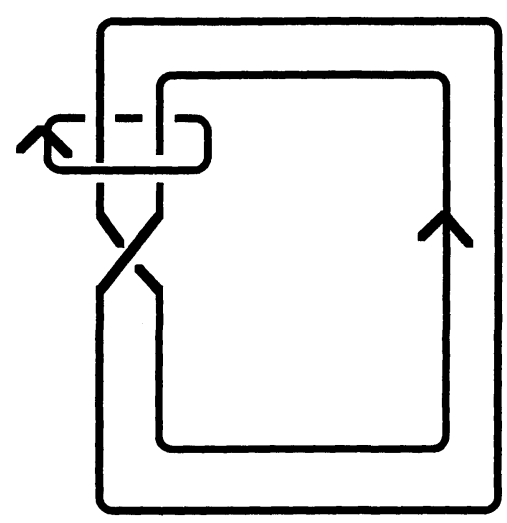

FIGURE 4c

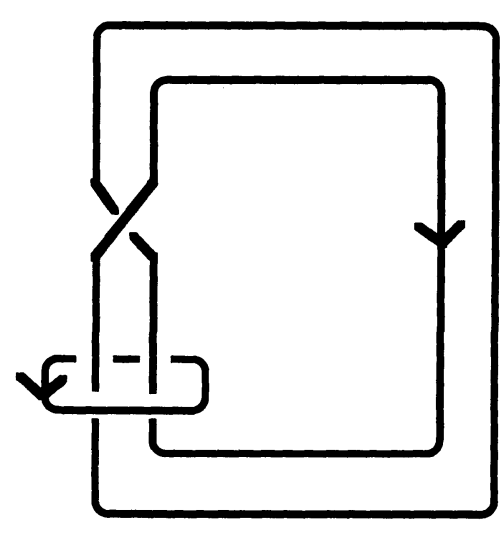

Figure 4d

Thus we have an annulus $H$ in $I \times Q$ with $H \cap(1 \times Q)=L\left(C_{\delta}\right)$ and $H \cap(0 \times Q)=L(D(\mathfrak{L}))$ where $\mathfrak{L}$ is the line with $L(\mathfrak{L})$ corresponding to the fiber over $[0,1, i] \in \Sigma$. We have a similar annulus for every point in $p_{k} \in R P(2)$ and local orientation at $p_{k}$ There are exactly two complex conjugate lines in $C P(2)$ through a given point $p \in R P(2)$ which are tangent to $\Sigma$. So we can do the same construction as above. Or we can use the $\mathrm{SO}(3)$ action described in Remark (4.3) to move $H$ around to the position we want. Thus given a collection of points $p_{k}$ with local orientations around them we can find annulus $\left\{H_{k}\right\}$ in $I \times Q$, so that $H_{k} \cap(1 \times Q)=L\left(C_{k}\right)$ and $H_{k} \cap(0 \times Q)=L\left(D\left(\mathfrak{L}_{k}\right)\right)$. By moving the $p_{k}$ a small amount we can guarantee that the $\mathfrak{L}_{k}$ are distinct from all the lines appearing in the $\mathfrak{L}_{m_{i}}$.

Using (2.6), we can change the $F_{i}$ a small amount inductively so that the $G_{i}$ and the $H_{k}$ are in general position, i.e. intersect transversely with no triple points. We will have $G_{i}$ intersect $H_{k}$ in $m_{i}$ points. Note that the choice of orientation at $p_{k}$ and thus of $H_{k}$ picks out one of the two complex lines through $p_{k}$ and tangent to $\Sigma$, say $H_{k}^{+}$. Suppose $A_{i}$ is dividing. A complex orientation of $A_{i}$ picks out one component of $C A_{i}-A_{i}$, say $C A_{i}^{+}$. The positive points of intersection between $G_{i}$ and $A_{k}$ come from the points of intersection of $\mathrm{CA}_{i}^{+}$and $\mathrm{H}_{k}^{+}$. The negative points of intersection come from points of intersection of $T\left(C A_{i}^{+}\right)$and $H_{k}^{+}$. Since all the points of intersection of $C A_{i}^{+}$and $H_{k}^{+}$take place in $I \times \widetilde{Q}$ and $\widetilde{Q}$ is a rational homology ball we have by (3.1):

$$
\begin{aligned}
C A_{i}^{+} \circ H_{k}^{+} & =\operatorname{lk}\left(L_{+}\left(A_{i}\right), L_{+}\left(C_{k}\right)\right)-\operatorname{lk}\left(L_{+}\left(\mathfrak{L}_{m_{i}}\right), L_{+}\left(D\left(\mathfrak{L}_{k}\right)\right)\right) \\
& =r\left(A_{i}, p_{k}\right)+m_{i} / 2
\end{aligned}
$$

and $T\left(C A_{i}^{+}\right) \circ H_{k}^{+}=-r\left(A_{i}, p_{k}\right)+m_{i} / 2$. Thus $G_{i} \circ H_{k}=2 r\left(A_{i}, p_{k}\right)$. 
We calculate $H_{k} \circ H_{k}$ similarly using linking numbers in $Q$. It is quite easy to see that two distinct $H_{k}$ 's will intersect in 2 points of opposite sign.

REMARK (6.5). The diffeomorphism $\phi$ of $\overline{h^{-1}(\delta)}$ with $\overline{h^{-1}(1-\delta)}$ given by the flow associated to $\nabla h^{2}$ can be seen as follows. First we identify $\overline{h^{-1}(\delta)}$ with $Q$ using the normal bundle projection $\overline{h^{-1}(\delta)} \rightarrow$ $\bar{\Sigma}=R P(2)$. Next identify $\overline{h^{-1}(1-\delta)}$ with $Q$ using the normal bundle projection $h^{-1}(1-\delta) \rightarrow R P(2)$. Here we are giving $\overline{h^{-1}(1-\delta)}$ the orientation it acquires as the boundary of $\overline{h^{-1}[\delta, 1-\delta]}$ and $\overline{h^{-1}(\delta)}$ the orientation it acquires as the boundary of $\overline{h^{-1}[0, \delta]}$. Then $\phi: Q \rightarrow$ $Q$ is orientation preserving. (It had better be as there are no orientation reversing diffeomorphisms by [Ru].) In the proof of (6.1), we showed $\phi$ sends a fiber of $\overline{h^{-1}(\delta)} \rightarrow \bar{\Sigma}$ to $L(\mathfrak{L})$. The identification of $\overline{h^{-1}(\delta)}$ with $Q$ is only well defined modulo $\mathscr{A}$ which extends over the disk bundle. Therefore $\phi$ is isotopic modulo $\mathscr{A}$ on the domain to the map $\mathscr{T}$ discussed in $\S 3$.

7. A new formulation of a theorem of Viro and Zvonilov. Let $C$ be a collection of disjoint oriented simple closed curves $\left\{C_{i}\right\}$ in $R P(2)$. Let $\mathfrak{C}$ be the number of ovals. A pair of ovals is called injective if one is nested in the interior of the other. An injective pair of ovals is called negative if they are homologous in the complement of a point interior to both of ovals. Otherwise the pair is called positive. If $C$ includes a 1 -sided curve, we say an oval $O$ is negative, if the oval is homologous to twice the 1-sided curve in the complement of a point interior to the oval. Otherwise it is called positive. Let $\Pi^{+}$denote the number of positive injective pairs, and $\Pi^{-}$denote the number of negative injective pairs. Let $\Lambda^{+}$denote the number of positive ovals, and $\Lambda^{-}$denote the number of negative ovals. If there is no 1 -sided curve, we set $\Lambda^{+}$and $\Lambda^{-}$to be zero. These notations are due to Rokhlin [R2]. Given a collection as above, in $\S 3$ we defined a pushed off collection $C^{\prime}$. The following proposition follows easily from (3.1).

Proposition (7.1).

$$
\operatorname{lk}\left(L(C), L\left(C^{\prime}\right)\right)=2 \mathfrak{C}+4\left(\Pi^{-}-\Pi^{+}\right)+2\left(\Lambda^{-}-\Lambda^{+}\right) .
$$

THEOREM (7.2) (Rokhlin). If $A$ is a nonsingular dividing curve in $R P(2)$ with one of its complex orientations, then for the collection $A$ : $\mathfrak{C}+2\left(\Pi^{-}-\Pi^{+}\right)+\left(\Lambda^{-}-\Lambda^{+}\right)=\left[m^{2} / 4\right]$. 
Proof. Let $L_{m}^{\prime}$ be a copy of $L_{m}$ pushed off with the given framing. By $(3.1), \operatorname{lk}\left(L_{m}, L_{m}^{\prime}\right)$ is zero. It is easy to see that $\operatorname{lk}\left(L(A), L\left(A^{\prime}\right)\right)-$ $\operatorname{lk}\left(L_{m}, L_{m}^{\prime}\right)=G \circ G$ for the cobordism of (6.1). By (6.1), $G \circ G^{\prime}=$ $\left[m^{2} / 2\right]$.

Theorem (7.3). Let $A_{1}$ and $A_{2}$ be two nonsingular dividing real algebraic curves which intersect transversely in $r$ points. Then

$$
\left|\operatorname{lk}\left(L\left(A_{1}\right), L\left(A_{2}\right)\right)\right| \leq\left(m_{1} m_{2}-r\right) / 2
$$

with equality modulo two.

Proof. We apply (6.2). Twice the total number of intersections of $G_{1}$ and $G_{2}$ is $m_{1} m_{2}-r$. Let $\tau$ denote twice the number of negative intersections. So $m_{1} m_{2}-r \geq \tau \geq 0$. Then $\operatorname{lk}\left(L\left(A_{1}\right), L\left(A_{2}\right)\right)=$ $G_{1} \circ G_{2}=\left(m_{1} m_{2}-r\right) / 2-\tau$.

Suppose $A_{1}$ is the zero locus of $F_{1}$ in $R P(2)$ and $A_{2}$ is the zero locus of $F_{2}$ in $R P(2)$. Then $A_{1} \cup A_{2}$ is the zero locus of $F_{1} \cdot F_{2}$ in $R P(2)$ and $C A_{1} \cup C A_{2}$ is the zero locus of $F_{1} \cdot F_{2}$ in $C P(2)$. Suppose we have already changed the coefficients of $F_{2}$ using (2.6) so that $C A_{1}$ intersects $C A_{2}$ transversely. According to Brussotti's Theorem (see [Gu, p. 12]), we can change the coefficients of $F_{1} \cdot F_{2}$ an arbitrarily small amount while keeping them real so that we can affect an arbitrary choice of smoothing at each real double point. If necessary we may then make a further small choice of coefficients using (2.6) so that the zero locus in $C P(2)$ is nonsingular. Let $F$ be the resulting polynomial, $A$ its zero locus in $R P(2)$, and $C A$ its zero locus in $C P(2)$. Near the complex double points of $C A_{1} \cup C A_{2}, C A$ is isotopic to the resolution obtained by replacing neighborhoods of the double point on $C A_{1}$ and $C A_{2}$ by an annulus which is compatible with the orientations on $C A_{1}$ and $C A_{2}$ given by the complex structure. Suppose we choose the smoothing at each double point to be compatible to the complex orientations of $A_{1}$ and $A_{2}$. Recall the choice of complex orientations comes from a choice of one half of $C A_{1}$ denoted $C A_{1+}$ and a choice of one half of $C A_{2}$ denoted by $C A_{2+} \cdot A$ will divide $C A$ if and only if $C A_{1+}$ intersects only $C A_{2+}$ and misses $T\left(C A_{2}\right)$. This will happen if and only if all the intersections between $G_{1}$ and $G_{2}$ are positive; i.e. the $\tau$ of the proof of (7.2) is zero. Thus we have proved: 
TheOREM (7.4). Let $A_{1}$ and $A_{2}$ be two nonsingular dividing real algebraic curves which intersect transversely in $r$ points. Suppose $A$ is formed as above by smoothing the intersections using some complex orientations on $A_{1}$ and $A_{2}$. We have that $A$ is dividing if and only if $L k\left(L\left(A_{1}\right), L\left(A_{2}\right)\right)=\left(m_{1} m_{2}-r\right) / 2$ using the chosen complex orientations on $A_{1}$ and $A_{2}$. In this case the orientation on $A$ induced by the complex orientations on $A_{1}$ and $A_{2}$, is a complex orientation for $A$.

REMARK (7.5). Now if $A$ is dividing with this complex orientation, it must satisfy (7.1). Just thinking about linking numbers, we can see that, if $A_{1}$ and $A_{2}$ also satisfy (7.1),A will satisfy (7.1) if and only if $L k\left(L\left(A_{1}\right), L\left(A_{2}\right)\right)=\left(m_{1} m_{2}-r\right) / 2$. Viro and Zvonilov's theorem (4.8) of [V] is thus equivalent to (7.2), (7.3) and (7.4).

8. Kirby calculus. In this section, we use the Kirby calculus [K], [A-K] to describe $X_{n}, Q, \widetilde{Q}$, and $L(A)$ in $Q$. We also use the calculus to show $\widetilde{Q}$ is the lens space $L(4,3)$ and $Q$ is $S^{3}$ modulo the quaternion eight subgroup with the orientation reversed. We give a new proof of the theorem of Massey and Kuiper that $C P(2)$ modulo complex conjugation is $S^{4}$.

Proposition (8.1). $X_{n}$ can be built with one 0-handle, one 1-handle and one 2-handle as prescribed in Figure 5a. Thus $\widetilde{Q}$ and $Q$ are given respectively by the framed links in Figures $5 \mathrm{~b}$ and $5 \mathrm{c}$ (or 5d).

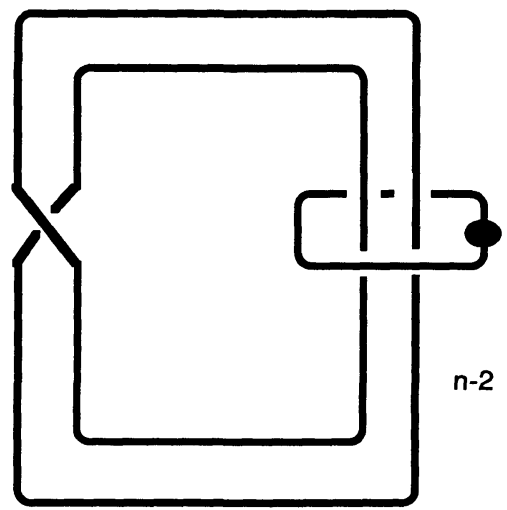

Figure 5a

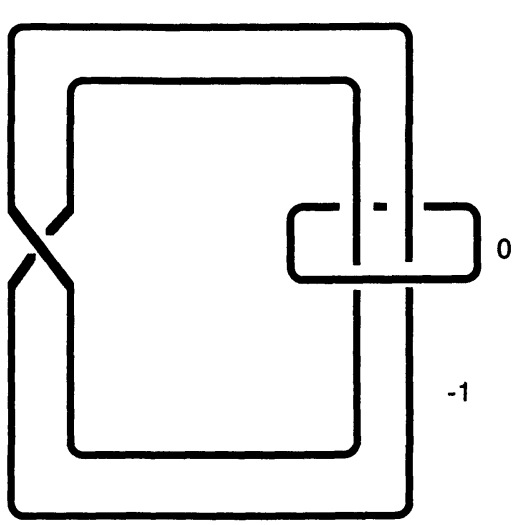

$\mathbf{R}$

FIGURE $5 b$ 


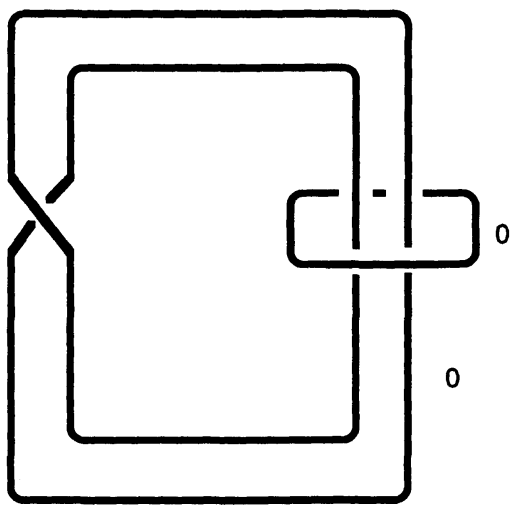

Q

FIGURE 5c

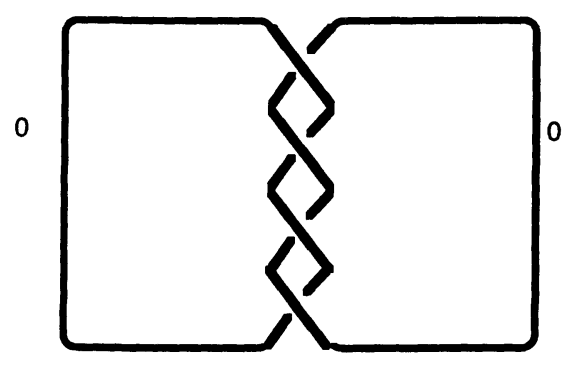

Q

Figure 5d

Proof. $R P(2)$ can be built with one 0 -handle, one 1-handle attached in a nonorientable way, and one 2 -handle. Since $X_{n}$ must be a trivial 2-disk bundle over each of these pieces, it can be built in the same way, except the one handle must be attached in an orientable way. Our first 0 -handle is just a 4-ball with boundary $S^{3}$. Our 1-handle is attached to $B^{4}$ along the two 3-balls pictured in Figure 6 in such a way that if the 3-ball is slid along the 1-handle, it will come out at the other 3-ball with the point labelled 1 landing at the point labelled $1^{\prime}$ on the other 3-ball, etc. Finally the 2-handle is attached along an $S^{1} \times D^{2}$ whose core circle consists of the arcs joining 4 to $1^{\prime}$ and 1 to $4^{\prime}$ in Figure 6 plus two arcs which travel straight over the 1-handle. We leave the framing of this last 2-handle temporarily undetermined. Finally note that the 0 -section of $X_{n}$ is isotopic to a disk bounding the unknot in $S^{3}$ pushed into $B^{4}$ union a 1-handle in the 4-dimensional 1-handle union the core of the last 2-handle. Now we isotope the picture so that the two three balls are identified by reflection in the plane separating them. See Figure 7a. Next following Akbulut and Kirby [A-K], we view $B^{4}$ union the 1-handle as the complement of an unknotted disk in $B^{4}$ and denote this by a circle with a dot. Figure $7 \mathrm{~b}$ then means: take $B^{4}$, excavate an open tubular neighborhood of the unknotted disk in $B^{4}$ which spans the dotted curve, and then attach a 2-handle to the other curve with framing still to be determined. The 0-section of $X_{n}$ is the evident Möbius band in the 3-sphere pushed into the 4ball union the core of the 2-handle. Since the framing on the attaching curve for the 2-handle which comes from the Möbius band is -2 , if we attach the 2-handle with framing -2 , we will get a bundle with euler number zero. Thus $X_{n}$ is obtained by attaching the 2-handle in 


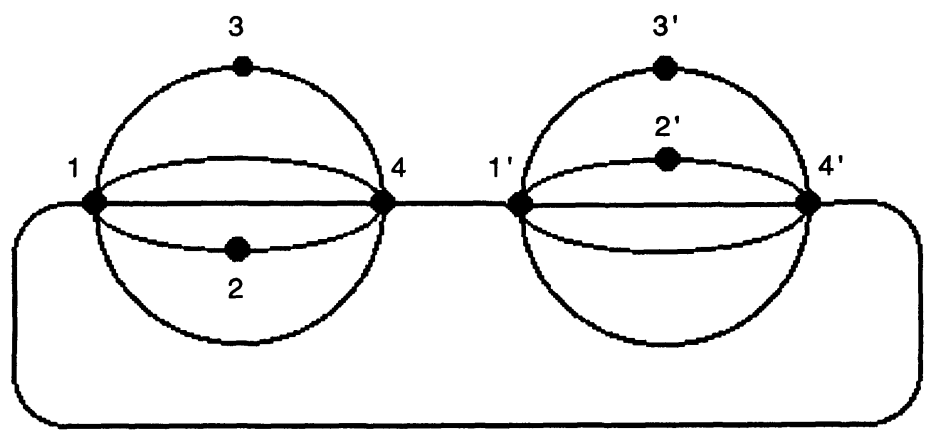

FIGURE 6

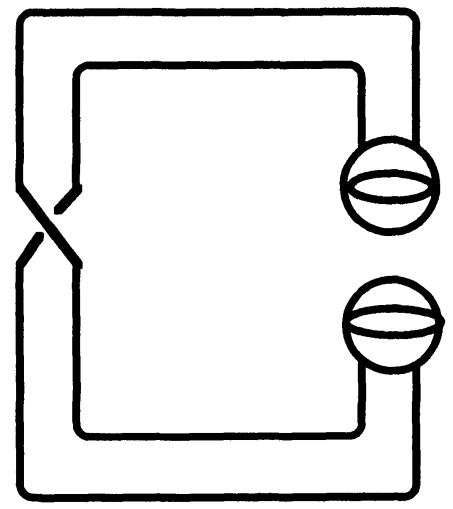

Figure 7a

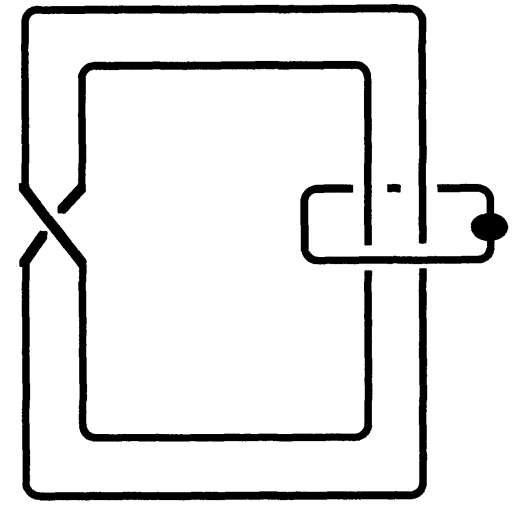

Figure $7 \mathrm{~b}$

Figure $7 \mathrm{~b}$ with framing $n-2$. To obtain a surgery description of $S_{n}$, we can just replace the circle with a dot with a 2-handle with framing zero as in Figures $5 b$ and $c$.

Proposition (8.2). $\widetilde{Q}$ is oriented diffeomorphic to $L(4,3)$ and $-Q$ is oriented diffeomorphic to $S^{3}$ thought of as the unit quaternions modulo the subgroup with element $\{ \pm 1, \pm 1, \pm j, \pm k\}$.

Proof. If we blow down the unknot which is framed -1 in the framed link description for $\widetilde{Q}$, we get an unknot with framing 4 . This is a description of $-L(4,1)=L(4,3)$. The framed link for $Q$ appears in Rolfsen [Ro, pp. 304-305] where he shows $\pi_{1}(Q)$ is the above quaternion eight group, and that $Q$ is the 3-fold branched cover of $S^{3}$ along the mirror image of the trefoil knot as drawn in Rolfsen's table. This is the link of the singularity $z^{3}+w^{2}=0$. Note the pictures of this link in both [Mi1] and [M] have the wrong orientation. 
The pictures in [E-N] have the correct orientation. Thus $Q$ is oriented diffeomorphic to the Breiskorn manifold $M(2,3,3)$ (oriented as the boundary of $z_{1}^{2}+z_{2}^{3}+z_{2}^{3}=\delta$ intersect a small ball around the origin) [Mi3, Lemma (1.1)]. By [Mi3, Theorem (4.5)], $M(2,3,3)$ is oriented diffeomorphic to $S^{3}$ modulo the left action of a discrete subgroup of $\mathrm{SU}(2)$ (the proof shows that the diffeomorphism is orientation preserving). By the calculation in Rolfsen this subgroup is isomorphic to $\{ \pm 1, \pm i, \pm j, \pm k\}$. It is not hard to see that any two subgroups of $\mathrm{SU}(2)$ isomorphic to $\{ \pm 1, \pm i, \pm j, \pm k\}$ must be conjugate. There is a well-known isomorphism of the unit quaternions with $\mathrm{SU}(2)$. However the left action of the unit quaternions on $S^{3}$ by left multiplication on itself is different than the action it gets from this isomorphism with $\mathrm{SU}(2)$. One action may be obtained from the other by conjugating with an orientation reversing involution on $S^{3}$. Therefore $-Q$ is diffeomorphic to the unit quaternions modulo $\{ \pm 1, \pm i, \pm j, \pm k\}$. Here we orient the unit quaternions as the boundary of the 4-disk.

REMARK (8.3). As the orientation preserving diffeomorphisms of $Q$ freely permute the non-zero elements of $H^{1}\left(Q, Z_{2}\right)$ (which classify the non-trivial 2-fold covers) all the non-trivial 2-fold covers of $M$ are oriented diffeomorphic to $\widetilde{Q}=L(4,3)$. As $L(4,3)$ does not possess an orientation reversing diffeomorphism (this follows easily from the linking form), $Q$ cannot possess an orientation reversing diffeomorphism. This is given another proof in [Ru]. It follows that although $S^{3}$ and $\mathrm{SU}(2)$ are isomorphic as Lie groups, no isomorphism between them matches up their usual left actions on $S^{3}$.

Now we wish to describe $L(C)$ in the above surgery description of $Q$, if $C$ is a collection of embedded curves in $R P(2)$. As $L(C)$ is determined up to isotopy by the isotopy class of $C$, we may isotope the 1-sided curve $\mathscr{L}$ (if any) so it consists of the core of 1-handle and part of the boundary of 0 -handle in the original handle decomposition of $R P(2)$. Isotope the remaining ovals into a small disk $D$ in the 0 handle. Lying over $\mathscr{L}$, there is a Klein bottle $K_{\mathscr{L}}$ and lying over each oval $C_{i}$, there is a torus $T_{i}$ sitting in the solid torus $T$ lying over $D$. If we follow these surfaces through our construction of $X_{2}$ and $Q, K_{\mathscr{L}}$ and $T$ wind up as pictured in Figure 8.

$L(\mathscr{L})$ is a section of $K_{\mathscr{L}} \rightarrow \mathscr{L}$. However there are only two sections up to isotopy in $K_{\mathscr{L}}$ and they differ by the antipodal map. Since the antipodal map restricted to $T$ is isotopic to the identity, we can pick the simplest looking section for $L(\mathscr{L})$ without loss of generality. 


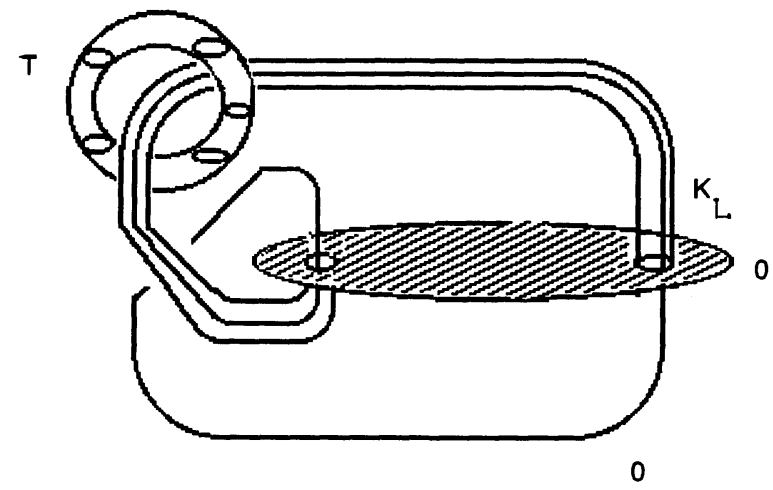

FIGURE 8

By definition $L\left(C_{i}\right)$ is a $(2,1)$ torus knot on $T_{i}$. Here the first factor tells us how many times the curve goes counterclockwise around the fiber direction in $T$, and the second number gives the degree of the projection onto $C_{i}$ (oriented counterclockwise). Note any complex orientation on $C_{i}$ is irrelevant here to the location of $L\left(C_{i}\right)$, but does then induce an orientation on $L\left(C_{i}\right)$ after its location is determined. This determines $L(C)$. For instance if $C$ is as pictured in Figure 9a then $L(C)$ is shown in 10b. In general $L(\mathscr{L})$ will appear as drawn and inside $T$, we will have a collection of $(2,1)$ torus knots lying over the different ovals of $C$. Note also that $L(\mathscr{L})$ by itself is isotopic to a meridian to the handle on the right so $L_{m}$ up to isotopy consists of $m$ unlinked meridians of this handle. See Remark (3.1). The process described in the proof of (6.3) allows one to see how $D(\mathscr{L})$ is obtained from $L(\mathscr{L})$ as well.

The surgery description of $Q$ given in Figure $5 \mathrm{~d}$ has an obvious symmetry of order 2 . This defines an involution which switches $f$ and $b$ in $H_{1}(Q)$. So this is isotopic to the involution called $\mathscr{T}$ in $\S 3$.

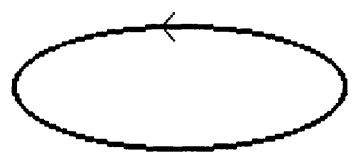

Figure 9a 


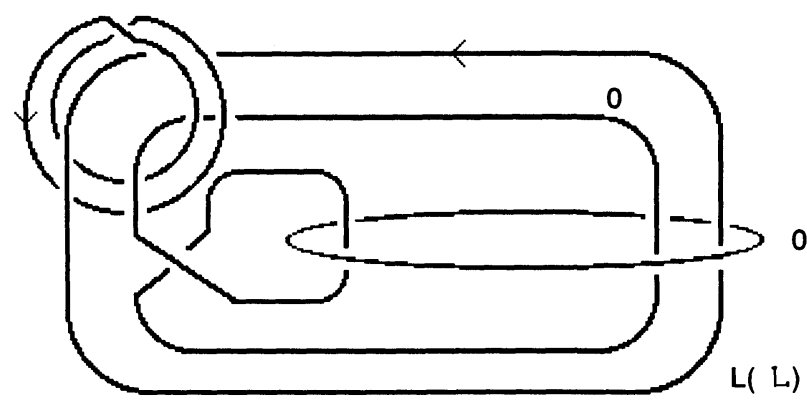

FIGURE 9b

Using Remark (6.5), we see that the orbit space $\overline{C P(2)}$ can be built from two copies of $X_{2}$ glued together by $\mathscr{T}$. This allows us to give a new proof of the following theorem.

TheOREM (8.4) (Massey [M], Kuiper [K], Arnold [A]). The orbit space of complex conjugation on $C P(2)$ is diffeomorphic to $S^{4}$.

Proof. Let $\mathscr{N}$ be a neighborhood of a fiber of $X_{2}$. It is of course diffeomorphic to $D^{2} \times D^{2}$. When glued to the second copy of $X_{2}$, it becomes a 2-handle attached along a meridian for the circle with dot in the handle decomposition of this second copy of $X_{2} . X_{2}-\operatorname{int}(\mathscr{N})$ is a $D^{2}$-bundle over a closed Möbius band with orientable total space. Thus $X_{2}-\operatorname{int}(\mathscr{N})$ is also a $D^{3}$ bundle over $S^{1}$ with orientable total space. So $X_{2}-\operatorname{int}(\mathscr{N}) \approx S^{1} \times D^{3}$ and can be built with a 0 -handle and a 1-handle. Thus our orbit space can be built with one 0-handle, one 1-handle, two 2-handles (one given by $\mathscr{N}$ ), a 3-handle and a 4-handle (turning upside down the decomposition of $X_{2}-\operatorname{int}(\mathscr{N})$ ). However the 2-handle given by $\mathscr{N}$ geometrically cancels the 1-handle, and thus our space has a decomposition with one 0 -handle, one 2-handle, one 3-handle and one 4-handle. Moreover the 2-handle is attached along an unknot with framing zero. Thus the orbit space is obtained by identifying the boundaries of $S^{2} \times D^{2}$ and $S^{1} \times D^{3}$. By [L-P], this must be the 4-sphere.

9. A generalization of the Tristram-Murasugi inequalities with an application. We begin by defining a common generalization of the Casson-Gordon invariant of a character on a closed oriented 3-manifold [C-G1], [C-G2], [Go, p. 42] and an invariant of $Z_{d}^{*}$-colored links in $S^{3}$ which we defined in our thesis [G1], [G2]. Let $M$ be a closed 
oriented 3-manifold (not necessarily connected), $L$ an oriented link in $M$ which comes equipped with a framing $f$ of its normal bundle, and $\psi$ a $d$-character (by this we mean an element in $H^{1}\left(M-L, Z_{d}\right)$ ). We insist that $\psi$ evaluated on meridians of $L$ be units. This last requirement is convenient but not really necessary, as we will show in a future paper. We will shortly see that in this situation we can always find a triple $(W, G, \theta)$ where $W$ is a 4-manifold with $\partial W=r M$ some multiple of $M, G$ is a properly embedded surface with $\partial G=r L$, and $\theta \in H^{1}\left(W-G, Z_{d}\right)$ where $\theta$ evaluates on meridians of $G$ to be units and $\theta$ restricted to each copy of $M$ on the boundary is $\psi$. In this situation, we will say $\partial(W, G, \theta)=r(M, L, \psi)$, that $(W, G, \theta)$ is a coboundary of $r(M, L, \psi)$ and that $(W, G, \theta)$ is a rational coboundary of $(M, L, \psi)$. Casson and Gordon showed that if $L$ is empty then $(M, L, \psi)$ has a rational coboundary with $G$ empty. Using $\theta$, we may construct $\widetilde{W}$, a branched cyclic cover of $W$ along $G$ with a specified generator $g$ for the group of covering transformations. Let $H$ denote the $\zeta=e^{2 \pi i / d}$ eigenspace for the action of $g$ on $\mathrm{H}_{2}(\widetilde{W}, C)$. For each component $G_{j}$ of $G$, let $a_{j}$ denote the integer in the range [0,d-1] which is congruent modulo $d$ to $\theta$ of a positive meridian to $G_{j}$. Let $\operatorname{Sign}(H)$ denote the signature of the intersection pairing on $\mathrm{H}_{2}(\widetilde{W}, R)$ Hermitianized and then restricted to $H$. Let $G^{\prime}$ denote a copy of $G$ made transverse to $G$ so that it has been pushed off along $L$ by the specified framings on the components of $L$. We define

$$
s(M, L, \theta)=\frac{1}{r}\left(\operatorname{Sign}(H)-\operatorname{Sign}(W)+\frac{2}{d^{2}} \sum_{j} a_{j}\left(d-a_{j}\right) G_{j} \circ G_{j}^{\prime}\right) .
$$

Proposition (9.1). Some multiple $(M, L, \theta)$ has a coboundary. $s(M, L, \theta)$ is well defined.

Proof. As $\psi$ on each meridian of $L$ gives a unit, we can find a framing $e$ on $L$ so that $\psi$ evaluates zero on push-offs of components of $L$ with this framing. Then $\psi$ extends uniquely to a characteristic $\psi^{\prime}$ on $e$-framed surgery to $M$ along $L$, which we denote $N$. Moreover if we let $V$ be the result of adding 2-handles to $M \times I$ along $L \times 0$ with the framing $e$ and $\Delta$ the union of the cores of the 2-handles with $L \times I$. There is a unique $\theta$ such that $(V, \Delta, \theta)$ is a coboundary of the disjoint union of $(-M, L, \psi)$ and $\left(N, \phi, \psi^{\prime}\right)$. Here $\phi$ denotes the empty set. As by Casson and Gordon, $\left(N, \phi, \psi^{\prime}\right)$ has a rational coboundary, it follows that $(M, L, \psi)$ has a rational coboundary. As 
the integers act on the framings of a component, we can define $b_{i}$ to be the "difference of" $e_{i}$ and $f_{i}$.

To see $s(M, L, \theta)$ is well defined we add multiples of two choices of rational coboundaries together to form a closed cyclic branched cover, use Novikov additivity, and apply some corollary of the $G$ Signature theorem, for instance (5.4) of [G2]. We omit the details of this by now familiar argument.

We also define $\eta(M, L, \psi)$ to be the rank of the $\zeta$-eigenspace for the action of $g$ on $H_{1}(\widetilde{M}, C)$ where $(\widetilde{M}, g)$ is the cyclic branched cover of $M$ along $L$ given by $\psi$. We call this the nullity. Actually this is the same as the rank of the $\zeta$-eigenspace for the action of $g$ on $H_{1}(M-L, C)$ where $(M-L, g)$ is the cyclic unbranched cover of $M-L$, by a Mayer-Vietoris argument [G2, (3.2)]. This generalizes $\eta(M, \psi)$ defined in [G2, p. 356]. We have $\eta(M, \phi, \psi)=\eta(M, \psi)$.

Proposition (9.2). $s(M, \phi, \psi)=\sigma(M, \psi)$, the Casson-Gordon invariant. Moreover, in general $s(M, L, \psi)=\sigma\left(N, \psi^{\prime}\right)+\operatorname{Sign} V-$ $\left(2 / d^{2}\right) \sum_{i} a_{i}\left(d-a_{i}\right) b_{i}$. Moreover $\eta(M, L, \psi)=\eta\left(N, \psi^{\prime}\right)$. Here $V$, $N, \psi^{\prime}$, and $b_{i}$ are as in the proof of (9.1) above.

Proof. We just use $(V, \Delta, \theta)$ to calculate $\partial(V, \Delta, \theta)$, and use the additivity of the invariant over disjoint union. The Mayer-Vietoris sequence argument gives the equality of nullities.

Using the above proposition and Theorem (3.6) of [G2], one may calculate $s(M, L, \psi)$ and $\eta(M, L, \psi)$ given a picture of $L$ in a surgery description of $M$. In $\S 8$, we gave such a description of $L(C)$ where $C$ is a collection of embedded curves in $R P(2)$. However it is quite laborious to calculate these invariants in this way. We are working on developing a new more efficient procedure.

If $M$ is a rational homology sphere we can assign a "canonical" framing to a link in one of two ways. The first way assigns to each component $K_{i}$ the framing for which when we take a push-off with that framing we get $K_{i}^{\prime}$ with $\operatorname{lk}\left(K_{i}, K_{i}^{\prime}\right)$ in the range $[0,1)$. If $\mathscr{L}$ denotes the $Q / Z$ valued linking form on $H_{1}(M)$, then this number is simply the representation of $\mathscr{L}\left(\left[K_{i}\right],\left[K_{i}\right]\right)$ in the range $[0,1)$. If $K_{i}$ is null homologous in $M$ (which it must be if $M$ happens to be a homology sphere), this framing is given by the way a Seifert surface for $K_{i}$ must meet $K_{i}$. For lack of a better name, we call this the first framing. The second framing is given similarly except we make $\operatorname{lk}\left(K_{i}, K_{i}^{\prime}\right)+\sum_{j \neq i} \operatorname{lk}\left(K_{i}, K_{j}\right)$ in the range $[0,1)$. If $\sum_{i}\left[K_{i}\right]$ is zero 
in $H_{1}(M)$, then $L$ has a Seifert surface and the second framing is given by the way a Seifert surface for $L$ meets $L$. Thus for links in a rational homology sphere, the extra data of a framing is really unnecessary to define an invariant. We simply define the invariant as above to be calculated with the first framing for instance. We have a slightly different invariant if we calculate with the second framing. Of course, they are related by a formula involving the linking number of the components. For our purposes, it is convenient to have links come equipped with the extra data of a framing. It may be the first framing, the second framing, or some other explicit framing like the framings defined in $\S 3$.

Note that if $M$ is a $Z_{d}$-homology sphere, then a character $\psi \in$ $H^{1}\left(M-L, Z_{d}\right)$ which evaluated on each meridian is a unit, is simply given by assigning freely to each component of $L$, a unit in $Z_{d}$. Thus for links in a $Z_{d}$-homology sphere, we have an invariant of $Z_{d}^{*}$ colored links. We will use the same letter $\psi$ to denote a coloring and the related character. In our thesis [G1], [G2, pg. 367], we define a signature invariant $\sigma(L, \psi)$ and nullity invariant $\eta(L, \psi)$ of colored links in $S^{3}$ generalizing the Levine-Tristram signatures of uncolored links [L], [T]. Later Cooper [C1], [C2] in his thesis defined a related signature invariant for $n$-component links in $S^{3}$ parameterized by an $n$-torus. More recently Smolinsky has defined a related invariant in high dimensions as well [S].

Proposition (9.3). Suppose $L$ is a link in $S^{3}$ with the second framing and $a_{i}$ is the color of the ith component of $L$. Then

$$
\begin{aligned}
& s\left(S^{3}, L, \psi\right)=\sigma(L, \psi)+\frac{2}{d^{2}} \sum_{i>j}\left(a_{i}-a_{j}\right)^{2} L k\left(K_{i}, K_{j}\right), \\
& \eta\left(S^{3}, L, \psi\right)=\eta(L, \psi) .
\end{aligned}
$$

Proof. Compare the formula in (9.2) with the definition of $\sigma(L, \psi)$ in [G2, middle of p. 367], and the definitions of the two nullity invariants.

When $L$ is nonempty, we will write $s(L, \psi)$ for $s(M, L, \psi)$ and $\eta(L, \psi)$ for $\eta(M, L, \psi)$, as $M$ is understood from $L$. Let $c(a, b)$ denote $-1+2(a+b) / d-4 a b / d^{2}$ for $a, b \in[1, d-1]$ representing units in $Z_{d}^{*}$.

Proposition (9.4). Let $L$ be the Hopflink in $S^{3}$ of two components with linking number one and the first framing. Suppose $\psi$ colors one 
component $a_{1}$ and the other $a_{2}$, where $0<a_{i}<d$. Then $s(L, \psi)=$ $c\left(a_{1}, a_{2}\right)$ and $\eta(L, \psi)$ is zero.

Proof. This follows from (9.3) above and (3.8) of [G2] which says $\sigma(L, \psi)=-1$. We only need to adjust for the difference between the first and second framing.

THEOREM (9.5). Let $d$ be a power of a prime $p$ and $M$ a $Z_{p^{-}}$ homology sphere. Let $\left\{L_{i}\right\}$ and $\left\{L_{i}^{\prime}\right\}$ be two collections of disjoint framed links in $M$ (we allow some of these links to be empty). For every $i$, let $G_{i}$ be an oriented link cobordism between $L_{i}$ and $L_{i}^{\prime}$. Assume the $G_{i}$ are transverse with no triple points in $I \times Q$. Let $G$ denote the union of the $G_{i}$ in $I \times Q, L$ the union of $\left\{L_{i}\right\}$ in $Q$ and $L^{\prime}$ the union of $\left\{L_{i}^{\prime}\right\}$. To simplify matters, we also assume that there exists a path on $G$ joining the two sides of $I \times Q$, and there are no closed components. Let $G_{i}^{\prime}$ denote $G_{i}$ pushed off itself so that along the boundary the push-off is given by the framings of the links. Color the cobordism by assigning $a_{i} \in Z_{d}^{*}$ to $G_{i}$. Let $\psi$ and $\psi^{\prime}$ denote the induced colorings of the links $L$ and $L^{\prime}$. We have the following inequality which is an equality modulo two;

$$
\begin{aligned}
& \mid s(L, \psi)-s\left(L^{\prime}, \psi^{\prime}\right)-\sum_{i>j} c\left(a_{i}, a_{j}\right) G_{i} \circ G_{j}-\frac{2}{d^{2}} \sum_{i} a_{i}\left(d-a_{i}\right) G_{i} \circ G_{i}^{\prime} \\
& \quad+\eta(L, \psi)+\eta\left(L^{\prime}, \psi\right) \leq \beta_{1}(G)-\beta_{0}(G)+2 \Delta
\end{aligned}
$$

where $\Delta=\operatorname{Minimum}\left\{\eta(L, \psi)+\eta\left(L^{\prime}, \psi\right),-1+\sum_{i} \beta_{0}\left(G_{i}\right)\right\}$.

Proof. Let $N$ denote a regular neighborhood of $G$ (the union of small tubular neighborhoods of the individual $\left.G_{i}\right), E=I \times Q-\operatorname{int}(N)$ and $S=N \cap E$. We have $H^{4}\left(I \times Q, E, Z_{p}\right) \approx H^{4}\left(N, S, Z_{p}\right) \approx$ $H_{0}\left(N, N \cap(\partial I \times Q), Z_{p}\right) \approx H_{0}\left(G, \partial G, Z_{p}\right) \approx 0$, as we hypothesized no closed components. Also

$$
\begin{aligned}
H_{1}\left(G, \partial G, Z_{p}\right) & \approx H_{1}\left(N, N \cap(\partial I \times Q), Z_{p}\right) \\
& \approx H^{3}\left(N, S, Z_{p}\right) \\
& \approx H^{3}\left(I \times Q, E, Z_{p}\right) \rightarrow H^{3}\left(I \times Q, Z_{p}\right)
\end{aligned}
$$

can be seen to be onto because there exists a path on $G$ joining the two sides of $I \times Q$. Thus using the long exact $Z_{p}$ cohomology sequence of the pair $(I \times Q, E)$, we see that $H_{3}\left(E, Z_{p}\right)=0$. Similarly $H_{2}\left(G, \partial G, Z_{d}\right) \approx H_{2}\left(N, N \cap(\partial I \times Q), Z_{d}\right) \approx H^{2}\left(N, S, Z_{d}\right) \approx$ $H^{2}\left(I \times Q, E, Z_{d}\right)$. As $H_{1}\left(I \times Q, Z_{d}\right)$ and $H_{2}\left(I \times Q, Z_{d}\right)$ are both zero, we see, using the same long exact sequence with $Z_{d}$ coefficients, 
that $H^{1}\left(E, Z_{d}\right) \approx H^{2}\left(I \times Q, E, Z_{d}\right) \approx H_{2}\left(G, \partial G, Z_{d}\right)$ which is a direct sum of a copy of $Z_{d}$ for each component of $G_{i}$. Moreover the character associated to a component of $G_{j}$ in this way is one on a positive meridian of that component, and zero on the meridians of other components of $G_{j}$ and the components of the other $G_{j}$. Thus we may pick $\Psi \in H^{1}\left(E, Z_{d}\right)$ which assigns $a_{i}$ to the meridians of each component of $G_{i}$ for all $i$. Note $\left.\Psi\right|_{1 \times Q}=\psi$ and $\left.\Psi\right|_{0 \times Q}=\psi^{\prime}$.

Let $E_{\Psi}$ denote the regular $Z_{d}$ cover of $E$ given by $\Psi$, together with the induced generator for the group of covering transformation, as in $\S 1$ of [G2]. We may complete $E_{\Psi}$ to form a space $\widehat{E_{\Psi}}$ lying over $I \times Q$ which is a branched cover of $I \times Q$ minus the points of intersection of the $G_{i}$ and above each of these points we have a single point which has a neighborhood which is a cone on the lens space which is the branched cover of $S^{3}$ along the Hopf link which describes the point of intersection. Let $W$ denote $I \times Q$ minus small open spherical neighborhoods of the points of intersection, and $\widetilde{W}$ the part of $\widehat{E_{\Psi}}$ which lies over $W$. Note we may use $(W, G \cap W, \Psi)$ to calculate $s(L, \psi)-s\left(L^{\prime}, \psi^{\prime}\right)-\sum s\left(H_{k}, \psi_{k}\right)$ where $k$ runs over all the points of intersection and $\left(H_{k}, \psi_{k}\right)$ denotes the Hopf link which describes the intersection together with the restriction of $\Psi$. Since $\operatorname{Sign} W=0$, we have that $s(L, \psi)-$ $s\left(L^{\prime}, \psi^{\prime}\right)-\sum s\left(H_{k}, \psi_{k}\right)$ is $\operatorname{Sign}(H)+\left(2 / d^{2}\right) \sum_{i} a_{i}\left(d-a_{i}\right) G_{i} \circ G_{i}^{\prime}$. By (9.4), $\sum s\left(H_{k}, \psi_{k}\right)=\sum_{i>j} c\left(a_{i}, a_{j}\right) G_{i} \circ G_{j}$. Here $H$ denotes the $\zeta$ eigenspce for the action on $H_{2}(\widetilde{W}, C)$ together with the restriction of the intersection Hermitian form. Thus we have that $\operatorname{Sign}(H)$ is given by $s(L, \psi)-s\left(L^{\prime}, \psi^{\prime}\right)-\sum_{i>j} c\left(a_{i}, a_{j}\right) G_{i} \circ G_{j}-\frac{2}{d^{2}} \sum_{i} a_{i}\left(d-a_{i}\right) G_{i} \circ G_{i}^{\prime}$. Let $\eta$ denote the nullity of this form.

We will let $\rho_{k}(X)$ denote $\operatorname{dim} H_{k}\left(X, Z_{p}\right), \bar{H}_{k}(Y)$ denote the $\zeta$ eigenspace for $g$ acting on $H_{k}(Y, C)$, where $Y$ denotes a space with $Z_{d}$ action with a specified generator $g$, and $\bar{\beta}_{k}(Y)$ denote the dimension of $\bar{H}_{k}(Y)$. Mayer-Vietoris sequences show $\bar{\beta}_{k}\left(E_{\Psi}\right)=\bar{\beta}_{k}\left(\widehat{E}_{\Psi}\right)=$ $\bar{\beta}_{k}(\widetilde{W})$ for $k \neq 0$. Since $\rho_{3}(E)=0$, by $[\mathbf{G 2},(1.4)], \bar{\beta}_{3}\left(\widehat{E_{\Psi}}\right)=0$. By $[G 2,(1.1)], \bar{\chi}(E)$, the alternating sum of the $\bar{\beta}_{k}(X)$, is $\chi(E)$. As $N$ deformation retracts on $G$, and $\chi(S)$ and $\chi(I \times Q)$ are zero, $\chi(E)=-\chi(G)=\beta_{1}(G)-\beta_{0}(G)$. As $E_{\Psi}$ is connected, $\bar{\beta}_{0}\left(E_{\Psi}\right)=0$. Thus $\bar{\chi}(E)=-\Omega+\bar{\beta}_{2}\left(\widehat{E_{\Psi}}\right)$ where $\Omega=\bar{\beta}_{1}\left(E_{\Psi}\right)=\bar{\beta}_{1}(\widetilde{W})$. Thus $\bar{\beta}_{2}\left(\widehat{E_{\Psi}}\right)=\beta_{1}(G)-\beta_{0}(G)+\Omega$. As $\rho_{1}(E)=\sum_{i} \beta_{0}\left(G_{i}\right), \Omega \leq-1+$ $\sum_{i} \beta_{0}\left(G_{i}\right)$, by $[\mathbf{G 2},(1.5)]$. By duality $\bar{\beta}_{1}(\widetilde{W}, \partial \widetilde{W})=\bar{\beta}_{3}(\widetilde{W})=0$. The first map in the exact sequence, $\bar{H}_{2}(\widetilde{W}, C) \rightarrow \bar{H}_{2}(\widetilde{W}, \partial \widetilde{W}, C) \rightarrow$ 
$\bar{H}_{1}(\partial \widetilde{W}, C) \rightarrow \bar{H}_{1}(\widetilde{W}, C) \rightarrow 0$, is given by a matrix which also represents the Hermitianized intersection pairing on $H$. As $\bar{\beta}_{1}(\partial \widetilde{W})=$ $\eta(L, \psi)+\eta\left(L^{\prime}, \psi\right)$, we have $\eta=\eta(L, \psi)+\eta\left(L^{\prime}, \psi\right)-\Omega$. From this it follows that $\Omega \leq \eta(L, \psi)+\eta\left(L^{\prime}, \psi\right)$. Thus we have $\Omega \leq \Delta$. Finally our main equality comes from $|\operatorname{Sign}(H)|+\eta \leq \bar{\beta}_{2}\left(\widehat{E_{\Psi}}\right)$ with equality mod two.

We now illustrate how this inequality may be applied to the cobordisms provided by $\S 6$. We will only apply this to the cobordism given by (6.1) although it is easy to apply it as well to those provided by (6.2) and (6.3). It is also worthwhile to apply this inequality to the cobordisms obtained by lifting the cobordisms in $I \times Q$ to $I \times \widetilde{Q}$ where $\widetilde{Q}$ is one of the five covering spaces of $Q$. In fact, the Viro-Zvonilov inequality (3.10) [V] may be obtained by applying these methods to the cobordism in $I \times \widetilde{Q}$.

Proposition (9.6). Let $d$ be an odd integer and $\psi$ be a $Z_{d}^{*}$-coloring of the components of $L_{r}$. Then $s\left(L_{r}, \psi\right)$ is zero and $\eta\left(L_{r}, \psi\right)=r-1$.

Proof. The diffeomorphism $\mathscr{T}$ of $Q$ sends $L_{r}$ to $r$ distinct fibers of the projection to $R P(2)$. We will compute the invariants of $\mathscr{T}\left(L_{r}\right)$ instead. Suppose $\mathscr{T}\left(L_{r}\right)$ lies over points say $\left\{p_{i}\right\}$ in $R P(2)$. As $R P(2)$ is a $Z_{d}$-homology disk, we may take the branched $Z_{d}$-cover of $R P(2)$ along the $p_{i}$ given by a character on the complement which sends the meridians to $a_{i}$. Denote this by $\widetilde{R P(2)}$. This extends to a branched cover of the associated disk bundle $X_{2}$ along the disk fibers over the $p_{i}$. The cover of the disk bundle equivariantly deformation retracts to $R \widetilde{P(2)}$. It is easy to see $\overline{\beta_{0}}(R \widetilde{P(2)})=\overline{\beta_{2}}(R \widetilde{P(2)})=$ 0 . Thus $\overline{\beta_{1}}(R \widetilde{P(2)})$ is minus $\bar{\chi}(R \widetilde{P(2)})$ which in turn is minus $\chi\left(R P(2)-\bigcup\left\{p_{i}\right\}\right)$ or $r-1$. The result follows.

The following theorem follows instantly from (6.1), (9.5) and (9.6). We have yet to work out an efficient method to calculate $s(L(C), \psi)$. However we are quite hopeful that when we do (9.7) will give new restrictions on the possible isotopy types of $C$.

THEOREM (9.7). Let $d$ be an odd prime power, and $b$ relatively prime to $d$ and in the range $[1, d-1]$. Suppose $A$ is a nonempty nonsingular dividing real algebraic curve of degree $m$ with its complex orientation. Give $L(A)$ the framing specified in $\S 3$, and the induced orientation, and let $\psi$ assign $b$ to every component of $L(A)$. Let [ ] 
denote the greatest integer function. Then

$$
\left|s(L(A), \psi)-\frac{2 b(d-b)\left[m^{2} / 2\right]}{d^{2}}\right|+\eta(L(A), \psi) \leq \frac{(m-1)(m-2)}{2} .
$$

\section{REFERENCES}

[A] V. I. Arnold, A branched covering of $C P(2) \rightarrow S^{4}$, hyperbolicity and projectivity topology, Siberian Math. J., 29 (1988), 717-726.

[AK] S. Akbulut and R. Kirby, Branched covers of surfaces in 4-manifolds, Math. Ann., 252 (1980), 111-113.

[B] R. Bott, Lectures on Morse theory, new and old, Bull. Amer. Math. Soc., 7, (1982), 331-358.

[CG1] A. Casson and C. McA. Gordon, Cobordism of classical knots, printed notes, Orsay 1975. (Reprinted in: ed A. Marin and L. Guillou, A la Recherche de la Topologie Perdue, Progress in Mathematics 62, Birkhauser, 1986.)

[CG2] _, On slice knots in dimension three, Proc. Symp. in Pure Math., XXX (1978), part two, pp. 39-53.

[C1] D. Cooper, Signatures of surfaces in 3-manifolds and applications to knot and link cobordism, thesis, Warwick University, 1982.

[C2] - The universal abelian cover of a link, 51-56, ed. R. Brown, and T. L. Thicksun, Low Dimensional Topology, L.M.S. 48 (1982), Cambridge University Press.

[E-N] D. Eisenbud and W. Neuman, Three-dimensional link theory and invariants of plane curve singularities, Annals of Math. Studies, 110 (1985), Princeton University Press.

[G1] P. Gilmer, Configurations of surfaces in 4-manifolds, Phd. thesis Berkeley, May 1978.

[G2] - Configurations of surfaces in 4-manifolds, Trans. Amer. Math. Soc., 264 (1981), 353-380.

[G3] __, Link cobordism in rational homology spheres, in preparation.

[G4] _ Real algebraic curves and link cobordism II, in preparation.

[Go] C. McA. Gordon, Aspects of Classical Knot Theory, Knot Theory, Lecture Notes in Math. vol. 685, Springer, New York, (1978), 1-60.

[Gu] D. A. Gudkov, The topology of real projective algebraic varieties, Russian Math. Surveys 29, 4 (1974), 1-79.

[F1] T. Fiedler, New congruences in the topology of real plane curves, Soviet Math. Dokl., 27 (1983), 566-568.

[F2] _-, Real points on complex plane curves, Math. Ann., 284 (1989), 267-284.

[F3] _ Additional inequalities in the topology of real plane algebraic curves, Math. USSR Iszvestia, 27 (1986), no. 1, 183-191.

[F4] __, Pencils of lines and the topology of real algebraic curves, Math. USSR Iszvestia, 21 (1983), 161-170.

[K] R. Kirby, A calculus for framed links in $S^{3}$, Invent. Math., 45 (1978), 35-56.

[Ku] N. H. Kuiper, The quotient space of $C P(2)$ by complex conjugation is the 4-sphere, Math. Ann., 208 (1974), 175-177.

[L] J. Levine, Knot cobordism groups in codimension two, Comment. Math. Helv., 44 (1969), 229-244.

[L-P] F. Laudenbach and V. Poenaru, A note on 4-dimensional handlebodies, Bull. Math. Soc. France, 100 (1972), 337-344. 
[Ma] W. Massey, The quotient space of the complex projective plane under conjugation is a 4-sphere, Geometriae Dedicata, 2 (1973), 371-374.

[M] D. Mumford, Algebraic Geometry I Complex Projective Varietes, Grundlehren der mathematishen Wissenschaften 221, 1976, Springer-Verlag.

[Mi1] J. Milnor, Singular points of complex hypersurfaces, Annals of Math. Studies 61.

[Mi2] _ Lectures on the h-cobordism theorem, (1985), Princeton University Press.

[Mi3] _ On the three dimensional Breiskorn manifolds $M(p, q, r)$, ed. L. P. Neuwirth, Knots, Groups, and 3-manifolds, papers dedicated to the memory of R. H. Fox, Annals of Math. Studies, 84 (1975), Princeton University Press.

[N] M. Namba, Geometry of Projective Algebraic Curves, (1984), Marcel Dekker, New York and Basel.

[P] T. Price, Homeomorphisms of quaternion space and projective planes in four space, J. Austral. Math. Soc., 23 (1977), 112-128.

[R1] V. A. Rokhlin, Complex orientations of real algebraic curves, Funct. Anal. Appl., 6 (1978), 77-89.

[R2] Complex topological characteristics of real algebraic curves, Russian Math. Surveys, 33:5 (1978), 85-98.

[Ro] D. Rolfsen, Knots and Links, (1976) Mathematical lecture series 7, Publish or Perish.

[Ru] J. H. Rubinstein, On 3-manifolds that have finite fundamental group and contain Klein bottles, Trans. Amer. Math. Soc., 251 (1979), 129-137.

[S] L. Smolinsky, A generalization of the Levine-Tristram link invariant, Trans. Amer. Math. Soc., 315, no. 1 (1989), 205-217.

[S-T] Seifert and Threfall, A Textbook of Topology, (1980), Academic Press.

[T] A. G. Tristram, Some cobordism invariants for links, Proc. Cambridge Philos. Soc., 66 (1969), 251-264.

[V1] O. Ya. Viro, Progress in the topology of real algebraic varieties over the last six years, Russian Math. Surveys, 41 (1986), 55-82.

[V2] L Real plane curves of degrees 7 and 8 : new prohibitions, Math. U.S.S.R. Iszvestia, 23 (1984), 409-422.

[W] G. Wilson, Hilbert's sixteenth problem, Topology, 17 (1978), 53-73.

Received August 20, 1990 and in revised form February 20, 1991.

Louisiana State UNIVERSITY

BATON Rouge, LA 70803 



\section{PACIFIC JOURNAL OF MATHEMATICS EDITORS}

V. S. VARADARAJAN

(Managing Editor)

University of California

Los Angeles, CA 90024-1555-05

Herbert Clemens

University of Utah

Salt Lake City, UT 84112

F. Michael Christ

University of California

Los Angeles, CA 90024-1555

THOMAS ENRIGHT

University of California, San Diego

La Jolla, CA 92093

\section{C. MOORE}

University of California

Berkeley, CA 94720

MARTIN SCHARLEMANN

University of California

Santa Barbara, CA 93106

HAROLD STARK

University of California, San Diego

La Jolla, CA 92093

STEVEN KERCKHOFF

Stanford University

Stanford, CA 94305

ASSOCIATE EDITORS

R. ARENS

E. F. BECKENBACH (1906-1982)

B. H. NeumanN

F. WOLF

(1904-1989)

K. YosHIDA

SUPPORTING INSTITUTIONS

UNIVERSITY OF ARIZONA

UNIVERSITY OF OREGON

UNIVERSITY OF BRITISH COLUMBIA

UNIVERSITY OF SOUTHERN CALIFORNIA

CALIFORNIA INSTITUTE OF TECHNOLOGY

UNIVERSITY OF CALIFORNIA

MONTANA STATE UNIVERSITY

STANFORD UNIVERSITY

UNIVERSITY OF NEVADA, RENO

UNIVERSITY OF HAWAII

NEW MEXICO STATE UNIVERSITY

OREGON STATE UNIVERSITY

UNIVERSITY OF UTAH

WASHINGTON STATE UNIVERSITY

UNIVERSITY OF WASHINGTON 


\section{Pacific Journal of Mathematics}

\section{Vol. 153, No. $1 \quad$ March, 1992}

Patrick Robert Ahern and Carmen Cascante, Exceptional sets for Poisson integrals of potentials on the unit sphere in $\mathbf{C}^{n}, p \leq 1 \ldots \ldots \ldots \ldots \ldots$

David Peter Blecher, The standard dual of an operator space ........... 15

Patrick Gilmer, Real algebraic curves and link cobordism .............. 31

Simon M. Goberstein, On orthodox semigroups determined by their

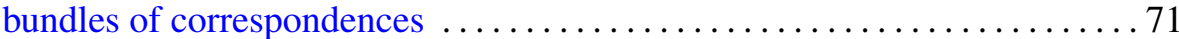

John Kalliongis and Darryl John McCullough, Homeotopy groups of irreducible 3-manifolds which may contain two-sided projective planes .......................................... 85

Yuji Konishi, Masaru Nagisa and Yasuo Watatani, Some remarks on actions of compact matrix quantum groups on $C^{*}$-algebras $\ldots \ldots \ldots \ldots 119$

Guojun Liao and Luen-Fai Tam, On the heat equation for harmonic maps from noncompact manifolds ........................... 129

John Marafino, Boundary behavior of a conformal mapping . .......... 147

Ji Min, A remark on the symmetry of solutions to nonlinear elliptic

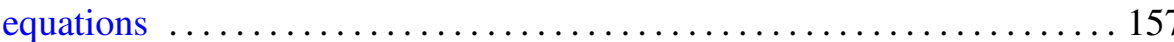

Paul Nevai and Walter Van Assche, Compact perturbations of orthogonal

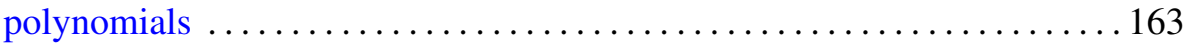

Kyril Tintarev, Level set maxima and quasilinear elliptic problems ....... 185 